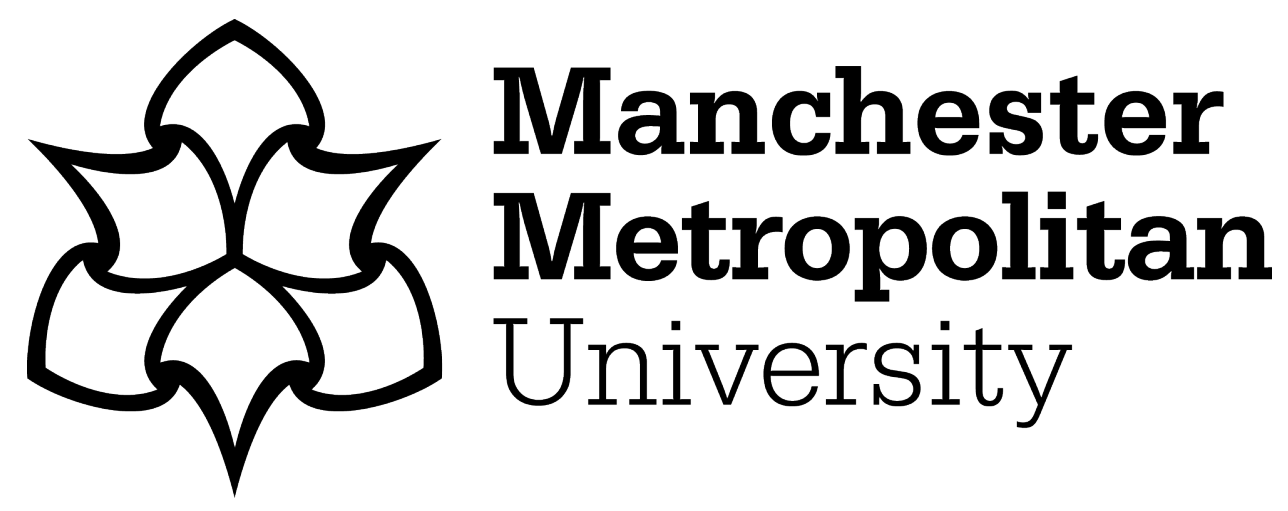

Zhou, Bingpeng, Liu, An, Lau, Vincent, Wen, Jinming, Mumtaz, Shahid, Bashir, Ali Kashif ORCID logoORCID: https://orcid.org/0000-0001-75952522 and Ahmed, Syed Hassan (2021) Performance Limits of Visible LightBased Positioning for Internet-of-Vehicles: Time-Domain Localization Cooperation Gain. IEEE Transactions on Intelligent Transportation Systems, 22 (8). pp. 5374-5388. ISSN 1524-9050

Downloaded from: https://e-space.mmu.ac.uk/625252/

Version: Accepted Version

Publisher: Institute of Electrical and Electronics Engineers (IEEE)

DOI: https://doi.org/10.1109/tits.2020.2974929

Please cite the published version 


\title{
Performance Limits of Visible Light-Based Positioning for Internet-of-Vehicle: Time-Domain Localization Cooperation Gain
}

\author{
Bingpeng Zhou, An Liu, Vincent Lau, Jinming Wen, Shahid Mumtaz, \\ Ali Kashif Bashir, and Syed Hassan Ahmed
}

\begin{abstract}
In this paper, we aim to give a unified performance limit analysis of the visible light-based positioning (VLP) for a vehicular user equipment (UE), which will help to understand the essence of time-domain localization cooperation and gain insights into how to improve the performance of the vehicular VLP system. This is not easy due to the complex system models and the complicated dependency between UE location performance and orientation performance. To achieve the above goal, we will first characterize the closed-form error bounds of the UE location and orientation at each time slot, respectively, in terms of Fisher information. Generally, the VLP error will propagate over time as the vehicular UE moves, and hence the VLP error at the current time slot is affected by the VLP performance at the previous time slot, the UE mobility and the channel quality. Based on the obtained VLP error bounds, we then reveal the impact of prior UE location knowledge, UE mobility and signal-to-noise-ratio on the VLP performance. Furthermore, the time-domain evolution of the VLP error is studied, where the convergence of the timedomain VLP error evolution is established and its closed-form stable state is quantified, which will shed light on the long-term performance of the vehicular VLP system.
\end{abstract}

Index Terms-Visible light-based positioning, long-term performance, CRLB, IOV, error propagation, mobile user tracking.

\section{INTRODUCTION}

$\mathbf{V}^{1}$ ISIBLE light-based positioning (VLP) is envisioned to be a promising solution to outdoor positioning, orientation and navigation for internet of vehicles (IOV), particularly in building-crowded areas where the global positioning system (GPS) sinal is blocked [5], [6]. For a vehicular user equipment (UE), the real-time tracking of UE location and orientation is particularly indispensable for location-aware IOV applications including intelligent transportation and automatic vehicle parking. Therefore, VLP has attracted increasing attention from

This work was supported by Science and Technology Plan of Shenzhen under Grant No. JCYJ20170818114014753.

Bingpeng Zhou is with Shenzhen Research Institute, Hong Kong University of Science and Technology (HKUST), Shenzhen, China, and he is also with the Department of Electronic and Computer Engineering, HKUST, Hong Kong 999077 (email:eebzhou@ust.hk).

An Liu is with the College of Information Science and Electronic Engineering, Zhejiang University, Hangzhou, China (email:anliu@zju.edu.cn).

Vincent Lau is with the Department of Electronic and Computer Engineering, HKUST, Hong Kong 999077 (email:eeknlau@ust.hk).

Jinming Wen is with the College of Information Science and Technology, Jinan University, Guangzhou, China (email:jinming.wen@mail.mcgill.ca).

Shahid Mumtaz is with the Instituto de Telecomunicações, P-3810-193 Aveiro, Portugal (email:smumtaz@av.it.pt).

Ali Kashif Bashir is with the Department of Computing and Mathematics, Manchester Metropolitan University, Manchester M13 9PL, UK (email:dr.alikashif.b@ieee.org).

Syed Hassan Ahmed is with the Department of Computer Science, Georgia Southern University, Statesboro, GA 30460, USA (email:sh.ahmed@ieee.org). industry and academia [1] and a number of VLP solutions have been proposed [4]-[16]. A detailed review of these solutions is given in [3] and [17].

In diverse VLP solutions, the received signal strength (RSS) of visible light signals is one popular measurement choice due to its simplicity and wide accessibility. The RSS-based VLP method uses LEDs as localization signal source (beacons) during indoor illumination, and it leverages the propagation knowledge of visible light signals with respect to (w.r.t.) UE location parameters to inversely determine the unknown UE location and orientation direction from RSS measurements. A lot of RSS-based VLP algorithms have been developed so far, for instance, in [4], [10], [11] and [13]. In this paper, we aim at providing a unified performance analysis on the RSS-based VLP for mobile UEs.

It is shown in [18] and [19] that the performance of signal propagation model-based localization methods (for instance, RSS-based VLP) is determined by the location-domain resolution of the propagation function and the additive measurement noise power, where the location-domain resolution indicates the sensitivity of UE location parameters w.r.t. measurement differences [20], [21]. Furthermore, the mobility of vehicular UE, prior location knowledge, the number of LEDs, prior UE location knowledge and signal-to-noise-ratio (SNR) will also affect the VLP performance. Hence, it is non-trivial to reveal the RSS-based VLP performance limits for vehicular UEs, which is indispensable for designing a location-aware IOV network and system resource allocation [22], as done in [23]-[27] for wideband system optimization.

It should be noted that, while the closed-form Cramer-Rao lower bound (CRLB) for wideband-based localization has been widely studied, e.g., in [20] and [28]-[31], the associated results cannot be directly applied to vehicular VLP due to the different physical propagation model. For instance, the visible light RSS is angular-sensitive, while the wideband signal is not. Hence, it is desirable to conduct a dedicated performance analysis for RSS-based vehicular VLP systems.

In the following, we shall first survey the related work on VLP performance analysis to motivate our own work, and then we will elaborate the contributions in this paper.

\section{A. Related Works}

In spite of early contributions to VLP performance analysis [8], [10], [32], [33], there are still several outstanding technical issues regarding the VLP performance at each time slot and 
the impact of some critical factors (such as SNR, UE mobility, prior knowledge), and thus the long-term VLP performance are not yet fully understood. These issues are elaborated below.

1) Single-Time-Slot VLP Performance: Vehicular VLP will determine the location parameters of a mobile UE at each time slot using the instantaneous RSS measurements. There are a number of works on the performance analysis of singletime-slot VLP performance [8], [10], [32]-[35]. For instance, in [10], the Cramer-Rao lower bound (CRLB) was studied for distance estimation error, where the LEDs are assumed with a downward orientation direction. Similarly, in [32], the CRLB on the ranging error with a known UE hight was derived. In addition to RSS, the performance limit of timeof-arrival-based VLP was studied in [8] and [33]. However, the required assumptions restrict the application of the above results in general cases, and the prior knowledge of UE location parameters from previous time slots (or GPS readings) is not studied in [8], [10] and [32]-[35]. In addition, these works focused on the ranging error or location error, but the extension to UE orientation estimate error is not trivial.

2) The Impact of UE Mobility: In contrast to static VLP, vehicular VLP is affected by UE mobility (unknown location shift and orientation variation), which can be viewed as a time-domain localization cooperation process, where the previous localization information will transfer over time and thus contribute to the UE location estimate at the next time slot. In this case, the UE mobility will affect how much prior knowledge from the previous time slot is inherited to the next time slot. Specifically, the UE mobility will dilute the strength of prior information from the previous time slot, and the previous location error as well as the UE orientation error will also propagate to the next-time-slot VLP phase [19]. As such, the VLP error will evolve over time, and the UE mobility and prior location information will eventually affect the longterm VLP performance. However, the dependency of the longterm VLP performance on UE mobility and prior UE location knowledge is not yet clear, and hence the investigation of timedomain VLP error evolution in the mobile UE tracking process is desirable and non-trivial.

\section{B. Contributions Of This Paper}

In this paper, we focus on the RSS-based VLP for vehicular UE tracking, and we aim to provide a unified framework for the performance analysis of time-domain VLP cooperation. Specifically, we seek to answer the following open questions.

- At each time slot, what performance can be achieved by the vehicular VLP system, given system resources, channel quality and UE mobility knowledge?

- How does the VLP error evolve over time, and how do we determine its long-term VLP performance limit?

- How do the UE mobility, channel quality and prior knowledge affect the long-term VLP performance?

These open questions will be answered via the closedform performance analysis, which is challenging due to the complex dependency of UE location performance and orientation performance on each other as well as the complex system functions. The obtained closed-form VLP error bounds build a theoretical foundation for the design of efficient VLP algorithms and VLP system optimization strategies. The contributions of this paper are summarized as follows.

- Closed-Form CRLB on VLP Error: We obtain the closedform CRLB on the instantaneous UE location error and orientation error, respectively, which quantifies the performance limit that any vehicular VLP algorithm can achieve. Unlike the existing works on the error bound of the transmission distance [8], [10], [33] or UE location [34], [35] with restrictive assumptions on the LED orientation direction and the UE height, (i) our results are straightforwardly related to the UE location CRLB and UE orientation CRLB without any restriction on the UE pose. In addition, (ii) we account for the prior UE location/orientation knowledge from GPS or inertial measurement unit (IMU) readings, and the information contribution of this prior knowledge to the RSS-based VLP performance is quantified. (iii) Our CRLB clearly shows the information contribution of prior knowledge, UE mobility and observation knowledge to the VLP performance at each time slot.

- Closed-Form Analysis of VLP Error Evolution: The timedomain error evolution (TDEE) of the vehicular VLP system is quantitatively analysed, which is challenging due to the complex TDEE function. In this paper, (iv) the convergence of the TDEE is established and (v) the closed-form stable state of TDEE is obtained, which sheds light on the long-term VLP performance limits. It is shown that the vehicular VLP error will converge downwards to a stable state when the observation information gain from LED sources is able to compensate the UE mobility-caused information reduction, and the stable state is dependent on the overall observation information, UE mobility and prior knowledge. In addition, (vi) the impact of UE mobility and prior knowledge on long-term VLP performance is analyzed.

- Guidelines for VLP System Optimization: Based on the obtained CRLBs, (vii) we give guidelines of vehicular VLP system optimization (e.g., LED power allocation, smart LED source selection, LED orientation steering) for localization error reduction, which is challenging due to the uncertain UE location parameters and the non-convex problem nature. Unlike the previous works in [22] and [35], which needs to solve a non-convex optimization problem over the UE location, we reconsider it as a stochastic optimization problem, which is convex w.r.t. the power allocation variable. This renders a low-cost power allocation solution, since the non-convex optimization in UE location is no longer required. The obtained guidelines will help to fundamentally improve the overall performance of the vehicular VLP system.

The remainder of this paper is organized as follows. Section II presents the system model. The closed-form CRLB is given in Section III. The TDEE is analysed in Section IV, and the effect of UE mobility is analysed in Section V. Guidelines are given in Section VI. Numerical results are presented in Section VII. We conclude our work in Section VIII. 


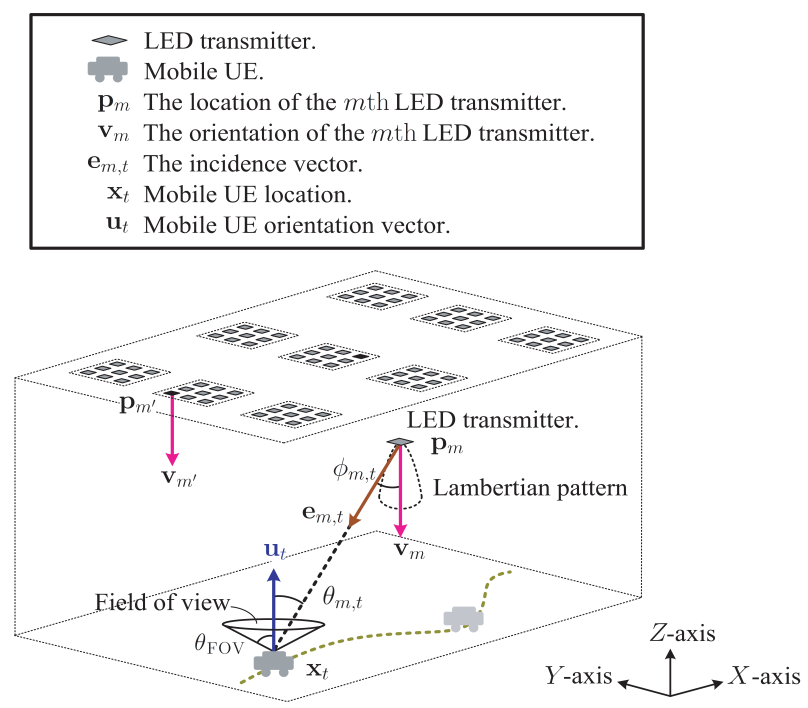

Fig. 1. Illustration of RSS-based VLP for mobile UE.

\section{System ModeL}

We first elaborate the VLP system setup, and then explicate the UE mobility model and measurement model, respectively.

\section{A. System Setup of VLP}

We consider a general three-dimensional vehicular VLP system with $M$ LED transmitters and one mobile UE receiver equipped with photodiodes, as illustrated in Fig. 1. We use $\mathbf{p}_{m} \in \mathbb{R}^{3}$ and $\mathbf{v}_{m} \in \mathbb{R}^{3}$ to denote the location and orientation vector of the $m$ th LED transmitter, respectively, for $m=1, \cdots, M$, where the LED orientation means its main emitting direction, as shown in Fig. 1. We assume $\left\|\mathbf{v}_{m}\right\|_{2}=1$ without loss of generality, where $\|\bullet\|_{2}$ is the $\ell_{2}$-norm on a vector. These LEDs will act as anchors for UE localization, and we assume their locations and orientation vectors are known. We also assume some visible light communication (VLC) protocols [36] (e.g., IEEE 802.15.7) and multiple access methods [37] (e.g., the time-division multiple access method) are well defined in the VLP system such that the signals from different LED sources are distinguishable.

\section{B. UE Location Model}

We assume the mobile UE randomly moves with a random orientation direction within the system area. Let $\mathbf{x}_{t}$ and $\mathbf{u}_{t} \in$ $\mathbb{R}^{3}$ denote the UE position and orientation vector, respectively, at time instant $t$, which are unknown parameters to estimate, where $t=1,2, \cdots$. We shall elaborate the prior knowledge and the mobility model of the UE, respectively.

1) Prior Location Knowledge: In practice, there might be prior knowledge of UE location parameters, for instance, from IMU readings, GPS or WiFi localization results, which could be exploited by practical VLP systems for improving the UE localization performance. Let $\widetilde{\mathbf{x}}_{t}$ and $\widetilde{\mathbf{u}}_{t} \in \mathbb{R}^{3}$ be the UE location and orientation readings, respectively, at the $t$ th time slot. In this paper, we generally assume the UE location $\mathrm{x}_{t}$ and orientation $\mathbf{u}_{t}$ follow a Gaussian distribution around their prior states $\widetilde{\mathbf{x}}_{t}$ and $\widetilde{\mathbf{u}}_{t}$, respectively:

$$
\begin{aligned}
& \mathbf{x}_{t} \sim \mathcal{N}\left(\mathbf{x}_{t} \mid \widetilde{\mathbf{x}}_{t}, \chi_{\text {prior }}\right), \\
& \mathbf{u}_{t} \sim \mathcal{N}\left(\mathbf{u}_{t} \mid \widetilde{\mathbf{u}}_{t}, \mathbf{U}_{\text {prior }}\right),
\end{aligned}
$$

where $\chi_{\text {prior }}$ and $\mathbf{U}_{\text {prior }} \in \mathbb{S}^{3}$ are the precision matrices of the prior location readings $\widetilde{\mathbf{x}}_{t}$ and $\widetilde{\mathbf{u}}_{t}$, respectively.

This above Gaussian prior model is reasonable due to the following reasons: (1) The Gaussian model is theoretically reasonable for large samples due to the central limit theorem [38]; (11) The Gaussian model renders tractable system modeling and easy theoretical analysis; (111) Given the mean and variance of the prior state, the Gaussian distribution gives rise to the maximum modeling entropy; i.e., the Gaussian assumption is the one with minimum restriction on the uncertainties of unknown parameters [39], [40]. Hence, the Gaussian model has the lowest risk in distribution modeling mismatch, if only given the mean and variance of prior UE location. It is shown in [41] and [42] that the unbiased estimation error can be approximated by a Gaussian random vector, for a high SNR. Hence, it is a common practice to model the estimation error as a Gaussian variable [31], [34], [35], [43], [44]. ${ }^{1}$

It should be note that our analysis can cover the case that there is no prior knowledge when $\chi_{\text {prior }}^{-1}$ and $\mathbf{U}_{\text {prior }}^{-1} \rightarrow \mathbf{0}_{3 \times 3}$.

2) Mobility Model: In practice, the vehicular UE may move either in a completely random manner or following a path possibly with a time-varying velocity. In order to establish a general VLP performance analysis framework, in this paper, we consider a general case that the UE movement direction is completely unknown at all time slots.

We assume the UE position $\mathrm{x}_{t}$ at the th time slot is transferred from the previous location $\mathbf{x}_{t-1}$ with a certain location shift $\wp_{t} \in \mathbb{R}^{3}$, i.e., $\mathbf{x}_{t}=\mathbf{x}_{t-1}+\wp_{t}$, where the UE location shift $\wp_{t}$ is assumed to be a zero-mean Gaussian process, i.e., $\wp_{t} \sim \mathcal{N}\left(\wp_{t} \mid \mathbf{0}_{3 \times 1}, \chi_{\text {trans }}\right)$ with $\chi_{\text {trans }} \in \mathbb{S}^{3}$ being the precision matrix (inverse variance) of the UE location transition. Specifically, the UE location $\mathbf{x}_{t}$ is a variable with a random transition from its previous state $\mathbf{x}_{t-1}$, i.e.,

$$
\mathbf{x}_{t} \sim \mathcal{N}\left(\mathbf{x}_{t} \mid \mathbf{x}_{t-1}, \chi_{\text {trans }}\right) .
$$

Similarly, for UE orientation $\mathbf{u}_{t}$, we assume

$$
\mathbf{u}_{t} \sim \mathcal{N}\left(\mathbf{u}_{t} \mid \mathbf{u}_{t-1}, \mathbf{U}_{\text {trans }}\right),
$$

where $\mathbf{U}_{\text {trans }} \in \mathbb{S}^{3}$ is the precision of UE orientation transition. For simplicity, we assume the location and orientation transition precisions are invariant over time. In addition, we consider a general case without any assumption on the UE movement direction, and hence we employ the above Gaussian process to model UE mobility for generality.

The transition precision parameters indicate the mobility of the UE. When the transition precision matrix $\chi_{\text {trans }}$ and $\mathbf{U}_{\text {trans }}$ tend to infinity, it means that there is no UE location transition, i.e., the UE location $\mathbf{x}_{t}$ and orientation $\mathbf{u}_{t}$ are invariant over

\footnotetext{
${ }^{1}$ For the non-Gaussian prior distributions of UE location parameters, it is common to use the Laplacian approximation method to extract its first-twoorders statistical characterizations [45], [46], which is equivalent to using the Gaussian model to locally approximate the non-Gaussian distribution. Hence, it is finally identical to our Gaussian prior model.
} 
time. On the contrary, when $\chi_{\text {trans }}$ and $\mathbf{U}_{\text {trans }}$ tend to zero, it means that the UE jumps unpredictably and it can appear anywhere with any orientation direction, i.e., $\mathbf{x}_{t}$ and $\mathbf{u}_{t}$ will not depend on their previous states at all.

\section{Measurement Model}

Visible light RSS used as measurements for VLP is dependent on LED emitting power, radiation, transmission distance, incidence angle gain and characteristic constants of UE receiver. A detailed explanation can be found in [47].

For notation ease, let $\mathbf{e}_{m, t}$ and $\rho_{m, t}$ denote the incidence vector and transmission distance, respectively, given by

$$
\begin{aligned}
\rho_{m, t} & =\left\|\mathbf{x}_{t}-\mathbf{p}_{m}\right\|_{2}, \\
\mathbf{e}_{m, t} & =\frac{\mathbf{x}_{t}-\mathbf{p}_{m}}{\rho_{m, t}}
\end{aligned}
$$

Furthermore, let $\phi_{m, t}$ be the irradiance angle, i.e., the angle between the $m$ th LED transmitter's orientation $\mathbf{v}_{m}$ and the incidence vector $\mathbf{e}_{m, t}$, as shown in Fig. 1, and let $\theta_{m, t}$ be the incidence angle between the receiver's orientation vector $\mathbf{u}_{t}$ and the incidence vector $\mathbf{e}_{m, t}$, given by

$$
\begin{aligned}
\phi_{m, t} & =\arccos \left(\frac{\left(\mathbf{x}_{t}-\mathbf{p}_{m}\right)^{\top} \mathbf{v}_{m}}{\left\|\mathbf{x}_{t}-\mathbf{p}_{m}\right\|_{2}}\right) \\
\theta_{m, t} & =\arccos \left(\frac{\left(\mathbf{p}_{m}-\mathbf{x}_{t}\right)^{\top} \mathbf{u}_{t}}{\left\|\mathbf{x}_{t}-\mathbf{p}_{m}\right\|_{2}\left\|\mathbf{u}_{t}\right\|_{2}}\right)
\end{aligned}
$$

where $\bullet^{\top}$ denotes the transpose. These geometrical propagation parameters will affect the RSS, as elaborated later.

Let $\mathrm{z}_{m, t}$ denote the visible light RSS associated with the $m$ th LED transmitter at time slot $t$, which can be characterized as the following general form,

$$
\mathrm{z}_{m, t}=h_{m}\left(\mathbf{x}_{t}, \mathbf{u}_{t}\right)+\epsilon_{m, t}, \forall m \in \Omega_{t},
$$

where $\epsilon_{m, t}$ is the measurement noise, which is assumed to follow a zero-mean Gaussian distribution with precision (inverse variance) $\omega$, i.e., $\epsilon_{m, t} \sim \mathcal{N}\left(\epsilon_{m, t} \mid 0, \omega\right)$ [48], [49], [50], and $\Omega_{t}$ is the set of active LEDs depending on the field of view (FOV) of LEDs and UE, given by $\Omega_{t}=\left\{m|| \frac{\theta_{m, t}}{\theta_{\mathrm{FOV}}} \mid \leq\right.$ $\left.1,\left|\frac{\phi_{m, t}}{\phi_{\mathrm{FOV}}}\right| \leq 1, \forall m=1: M\right\}$, where $\theta_{\mathrm{FOV}}$ and $\phi_{\mathrm{FOV}}$ is the known FOV of the UE and LEDs, respectively.

In addition, $h_{m}\left(\mathbf{x}_{t}, \mathbf{u}_{t}\right)$ denotes the measurement function of visible light RSS, given by [5], [51]

$$
h_{m}\left(\mathbf{x}_{t}, \mathbf{u}_{t}\right)=\Psi_{R} \frac{(r+1)\left(\cos \left(\phi_{m, t}\right)\right)^{r} \cos \left(\theta_{m, t}\right)}{\left\|\mathbf{x}_{t}-\mathbf{p}_{m}\right\|_{2}^{2}},
$$

where $\Psi_{R}$ is a constant depending on LED emitting power, UE photodiode aperture, optical filter gain and optical concentrator gain [47], [11], while $r$ denotes the LED Lambertian order. In this paper, we only consider the line-of-sight (LOS) channel, due to the less diffuse scattering effect in VLCs [6], [11].

Given the relationship between $\left\{\theta_{m, t}, \phi_{m, t}\right\}$ and $\left\{\mathbf{x}_{t}, \mathbf{u}_{t}\right\}$ in (7) and (8), $h_{m}\left(\mathbf{x}_{t}, \mathbf{u}_{t}\right)$ can be rewritten as a function explicitly dependent on $\mathbf{x}_{t}$ and $\mathbf{u}_{t}$, i.e.,

$$
h_{m}\left(\mathbf{x}_{t}, \mathbf{u}_{t}\right)=\Psi_{R} \frac{(r+1)\left(\left(\mathbf{x}_{t}-\mathbf{p}_{m}\right)^{\top} \mathbf{v}_{m}\right)^{r}\left(\mathbf{p}_{m}-\mathbf{x}_{t}\right)^{\top} \mathbf{u}_{t}}{\left(\left\|\mathbf{x}_{t}-\mathbf{p}_{m}\right\|_{2}\right)^{r+3}\left\|\mathbf{u}_{t}\right\|_{2}} .
$$

Let $\mathbf{z}_{t}=\operatorname{vec}\left[\mathbf{z}_{m, t} \mid \forall m \in \Omega_{t}\right] \in \mathbb{R}^{\left|\Omega_{t}\right|}$ be the collection of measurements, where $\left|\Omega_{t}\right|$ is the size of set $\Omega_{t}$. Then, $\mathbf{z}_{t}$ can be modeled as the following linear function w.r.t. $\mathbf{u}_{t}$,

$$
\mathbf{z}_{t}=\mathbf{h}\left(\mathbf{x}_{t}, \mathbf{u}_{t}\right)+\boldsymbol{\epsilon}_{t},
$$

where $\mathbf{h}\left(\mathbf{x}_{t}, \mathbf{u}_{t}\right) \in \mathbb{R}^{\left|\Omega_{t}\right|}=\operatorname{vec}\left[h_{m}\left(\mathbf{x}_{t}, \mathbf{u}_{t}\right) \mid \forall m \in \Omega_{t}\right]$, and $\boldsymbol{\epsilon}_{t}$ is the noise vector.

\section{Problem Formulation of VLP}

The visible light RSS-based VLP is to estimate UE position $\mathbf{x}_{t}$ and orientation $\mathbf{u}_{t}$ from RSS measurement $\mathbf{z}_{1: t}$ and inertial measurements $\left\{\tilde{\mathbf{x}}_{1: t}, \tilde{\mathbf{u}}_{1: t}\right\}$, which can be achieved via the following maximization problem,

$$
\mathcal{P}_{\mathrm{VLP}}:\left(\hat{\mathbf{x}}_{t}, \hat{\mathbf{u}}_{t}\right)=\underset{\mathbf{x}_{t}, \mathbf{u}_{t}}{\arg \max } p\left(\mathbf{x}_{t}, \mathbf{u}_{t} \mid \mathbf{z}_{1: t}, \widetilde{\mathbf{x}}_{1: t}, \widetilde{\mathbf{u}}_{1: t}\right),
$$

where $\bullet_{1: t}=\left\{\bullet_{1}, \cdots, \bullet_{t}\right\}$, and $p\left(\mathbf{x}_{t}, \mathbf{u}_{t} \mid \mathbf{z}_{1: t}, \widetilde{\mathbf{x}}_{1: t}, \widetilde{\mathbf{u}}_{1: t}\right)$ is the posterior distribution at the $t$ th time slot, given by (13). Specifically, $p\left(\mathbf{z}_{t} \mid \mathbf{z}_{1: t-1}, \widetilde{\mathbf{x}}_{1: t-1}, \widetilde{\mathbf{u}}_{1: t-1}\right)$ is a normalizing constant given by (14), and $p\left(\mathbf{x}_{t}, \mathbf{u}_{t} \mid \widetilde{\mathbf{x}}_{t}, \widetilde{\mathbf{u}}_{t}\right)$ is the piror function from IMU or GPS readings, given by

$$
p\left(\mathbf{x}_{t}, \mathbf{u}_{t} \mid \tilde{\mathbf{x}}_{t}, \widetilde{\mathbf{u}}_{t}\right)=\underbrace{\mathcal{N}\left(\mathbf{x}_{t} \mid \widetilde{\mathbf{x}}_{t}, \boldsymbol{\chi}_{\text {prior }}\right) \mathcal{N}\left(\mathbf{u}_{t} \mid \widetilde{\mathbf{u}}_{t}, \mathbf{U}_{\text {prior }}\right)}_{\text {Inertial prior model }},
$$

while $p\left(\mathbf{x}_{t}, \mathbf{u}_{t} \mid \mathbf{z}_{1: t-1}, \widetilde{\mathbf{x}}_{1: t-1}, \widetilde{\mathbf{u}}_{1: t-1}\right)$ is the prediction function given by (16), and $p\left(\mathbf{z}_{t} \mid \mathbf{x}_{t}, \mathbf{u}_{t}\right)$ is the likelihood function,

$$
p\left(\mathbf{z}_{t} \mid \mathbf{x}_{t}, \mathbf{u}_{t}\right)=\prod_{m \in \Omega_{t}} \mathcal{N}\left(\mathrm{z}_{m, t} \mid h_{m}\left(\mathbf{x}_{t}, \mathbf{u}_{t}\right), \omega\right)
$$

Therefore, once the previous-time-slot posterior distribution $p\left(\mathbf{x}_{t-1}, \mathbf{u}_{t-1} \mid \mathbf{z}_{1: t-1}, \widetilde{\mathbf{x}}_{1: t-1}, \widetilde{\mathbf{u}}_{1: t-1}\right)$ is given, the current-timeslot posterior function $p\left(\mathbf{x}_{t}, \mathbf{u}_{t} \mid \mathbf{z}_{1: t}, \widetilde{\mathbf{x}}_{1: t}, \widetilde{\mathbf{u}}_{1: t}\right)$ can be determined by using the above Bayesian filtering rules [52], and finally the current posterior estimate $\hat{\mathbf{x}}_{t}$ and $\hat{\mathbf{u}}_{t}$ can be obtained via the maximization problem in (12).

In the following, we shall characterize the error bounds for the above Bayesian model-based VLP problem.

\section{ERROR BOUND OF VEHICULAR VLP}

The CRLB quantifies the minimum error that an unbiased estimator can achieve [38]. We adopted it as as a performance metric to benchmark the VLP errors. In this section, we shall reveal the closed-form CRLBs on the UE location and the orientation estimation errors, respectively, at each time slot.

\section{A. Closed-Form CRLB}

Let $\hat{\mathbf{x}}_{t}$ and $\hat{\mathbf{u}}_{t}$ be the UE location and orientation estimates, respectively, as per problem $\mathcal{P}_{\text {VLP. }}$. Based on Bayesian theory [38], at time slot $t$, given measurement $\mathbf{z}_{t}$, the UE location and orientation estimate errors are bounded from below as follows,

$$
\begin{aligned}
& \mathbb{E}_{\mathbf{z}_{t}}\left\{\left\|\hat{\mathbf{x}}_{t}-\mathbf{x}_{t}\right\|_{2}^{2}\right\} \geq \operatorname{trace}\left(\mathcal{B}_{\mathbf{x}_{t}}\left(\mathbf{x}_{t}, \mathbf{u}_{t}\right)\right), \\
& \mathbb{E}_{\mathbf{z}_{t}}\left\{\left\|\hat{\mathbf{u}}_{t}-\mathbf{u}_{t}\right\|_{2}^{2}\right\} \geq \operatorname{trace}\left(\mathcal{B}_{\mathbf{u}_{t}}\left(\mathbf{x}_{t}, \mathbf{u}_{t}\right)\right),
\end{aligned}
$$

where $\mathcal{B}_{\mathbf{x}_{t}}\left(\mathbf{x}_{t}, \mathbf{u}_{t}\right)$ and $\mathcal{B}_{\mathbf{u}_{t}}\left(\mathbf{x}_{t}, \mathbf{u}_{t}\right) \in \mathbb{S}^{3}$ denote the CRLBs on the UE location estimate error and the UE orientation estimate error, respectively. 


$$
p\left(\mathbf{x}_{t}, \mathbf{u}_{t} \mid \mathbf{z}_{1: t}, \widetilde{\mathbf{x}}_{1: t}, \widetilde{\mathbf{u}}_{1: t}\right)=\frac{p\left(\mathbf{z}_{t} \mid \mathbf{x}_{t}, \mathbf{u}_{t}\right) p\left(\mathbf{x}_{t}, \mathbf{u}_{t} \mid \mathbf{z}_{1: t-1}, \widetilde{\mathbf{x}}_{1: t-1}, \widetilde{\mathbf{u}}_{1: t-1}\right) p\left(\mathbf{x}_{t}, \mathbf{u}_{t} \mid \widetilde{\mathbf{x}}_{t}, \widetilde{\mathbf{u}}_{t}\right)}{p\left(\mathbf{z}_{t} \mid \mathbf{z}_{1: t-1}, \widetilde{\mathbf{x}}_{1: t-1}, \widetilde{\mathbf{u}}_{1: t-1}\right)}
$$

$$
p\left(\mathbf{z}_{t} \mid \mathbf{z}_{1: t-1}, \widetilde{\mathbf{x}}_{1: t-1}, \widetilde{\mathbf{u}}_{1: t-1}\right)=\iint p\left(\mathbf{z}_{t} \mid \mathbf{x}_{t}, \mathbf{u}_{t}\right) p\left(\mathbf{x}_{t}, \mathbf{u}_{t} \mid \mathbf{z}_{1: t-1}, \tilde{\mathbf{x}}_{1: t-1}, \widetilde{\mathbf{u}}_{1: t-1}\right) \mathrm{d}\left(\mathbf{x}_{t}, \mathbf{u}_{t}\right)
$$

$$
p\left(\mathbf{x}_{t}, \mathbf{u}_{t} \mid \mathbf{z}_{1: t-1}, \widetilde{\mathbf{x}}_{1: t-1}, \widetilde{\mathbf{u}}_{1: t-1}\right)=\underbrace{\iint p\left(\mathbf{x}_{t-1}, \mathbf{u}_{t-1} \mid \mathbf{z}_{1: t-1}, \widetilde{\mathbf{x}}_{1: t-1}, \widetilde{\mathbf{u}}_{1: t-1}\right) \mathcal{N}\left(\mathbf{x}_{t} \mid \mathbf{x}_{t-1}, \boldsymbol{\chi}_{\text {trans }}\right) \mathcal{N}\left(\mathbf{u}_{t} \mid \mathbf{u}_{t-1}, \mathbf{U}_{\text {trans }}\right) \mathrm{d}\left(\mathbf{x}_{t-1}, \mathbf{u}_{t-1}\right)}_{\text {Prediction model of UE mobility }} .
$$

We shall establish the closed-form expressions of the above CRLBs, which will show the informative contribution of prior knowledge, UE mobility and observation knowledge to the overall VLP performance at one time slot.

Theorem 1 (One-Time-Slot CRLB): At the th time slot, the CRLBs $\mathcal{B}_{\mathbf{x}_{t}}\left(\mathbf{x}_{t}, \mathbf{u}_{t}\right)$ and $\mathcal{B}_{\mathbf{u}_{t}}\left(\mathbf{x}_{t}, \mathbf{u}_{t}\right)$ of UE location and orientation, respectively, are given by

$$
\begin{aligned}
& \mathcal{B}_{\mathbf{x}_{t}}\left(\mathbf{x}_{t}, \mathbf{u}_{t}\right)=(\underbrace{\operatorname{SNR} \cdot \mathcal{D}_{\mathbf{x}_{t}}\left(\mathbf{x}_{t}, \mathbf{u}_{t}\right)}_{\text {Observation information } \mathcal{H}_{\mathbf{x}_{t}}^{\text {obs }}}+\boldsymbol{\chi}_{\text {pred }, t}^{\circ})^{-1}, \\
& \underbrace{\text { Observation information }}_{\text {Total location information } \mathcal{J}_{\mathbf{x}_{t}}\left(\mathbf{x}_{t}, \mathbf{u}_{t}\right)} \\
& \mathcal{B}_{\mathbf{u}_{t}}\left(\mathbf{x}_{t}, \mathbf{u}_{t}\right)=(\underbrace{\mathrm{SNR} \cdot \mathcal{D}_{\mathbf{u}_{t}}\left(\mathbf{x}_{t}, \mathbf{u}_{t}\right)}_{\text {Observation information } \mathcal{H}_{\mathbf{u}_{t}}^{\text {obs }}}+\mathbf{U}_{\text {pred }, t}^{\circ})^{-1}, \\
& \underbrace{\text { Observation information } \mathcal{H}_{\mathbf{u}_{t}}^{\text {obs }}} \\
& \text { Total location information } \mathcal{J}_{\mathbf{u}_{t}}\left(\mathbf{x}_{t}, \mathbf{u}_{t}\right)
\end{aligned}
$$

where SNR is given by SNR $=\omega \Psi_{R}^{2}, \chi_{\text {pred }, t}^{\circ}$ and $\mathbf{U}_{\text {pred }, t}^{\circ}$ are the overall prediction information (i.e., time-domain localization cooperation gain from time recursion and prior readings) of the UE location and orientation, respectively, given by (22) and (23), while $\mathcal{H}_{\mathbf{x}_{t}}^{\text {obs }}$ and $\mathcal{H}_{\mathbf{u}_{t}}^{\text {obs }}$ are the observation information (i.e., spatial-domain localization cooperation gain from LED sources $^{2}$ ) of the UE location and orientation, respectively, in which $\mathcal{D}_{\mathbf{x}_{t}}\left(\mathbf{x}_{t}, \mathbf{u}_{t}\right)$ and $\mathcal{D}_{\mathbf{u}_{t}}\left(\mathbf{x}_{t}, \mathbf{u}_{t}\right) \in \mathbb{S}^{3}$ are resolution information matrices given by (24) and (25), respectively.

Proof: See the proof in APPENDIX A.

For ease of notation, let $\boldsymbol{\alpha}_{t} \in \mathbb{R}^{6}=\left[\mathbf{x}_{t} ; \mathbf{u}_{t}\right]$ denote the joint variable of unknown UE location and orientation. The prediction information and the resolution information in the above theorem are explicated, respectively, as follows.

1) Prediction Information: The overall prediction information matrices $\boldsymbol{\chi}_{\text {pred }, t}^{\circ}$ and $\mathbf{U}_{\text {pred }, t}^{\circ}$ are given by

$$
\chi_{\text {pred }, t}^{\circ}=\underbrace{\chi_{\text {prior }}+\underbrace{\left(\chi_{\text {trans }}^{-1}+\mathcal{B}_{\mathbf{x}_{t-1}}\left(\boldsymbol{\alpha}_{t-1}\right)\right)^{-1}}_{\text {Prediction information } \boldsymbol{\chi}_{\text {pred }, t}}}_{\text {Overall prediction information of UE location }}
$$

\footnotetext{
${ }^{2}$ In our paper, we slightly abuse "spatial-domain localization cooperation" without ambiguity to represent the localization cooperation among LED sources, which is different from the concept of the conventional spatial-domain localization cooperation among UE agents.
}

$$
\mathbf{U}_{\text {pred }, t}^{\circ}=\underbrace{\mathbf{U}_{\text {prior }}+\underbrace{\left(\mathbf{U}_{\text {trans }}^{-1}+\mathcal{B}_{\mathbf{u}_{t-1}}\left(\boldsymbol{\alpha}_{t-1}\right)\right)^{-1}}_{\text {Prediction information } \mathbf{U}_{\text {pred } t}}}_{\text {Overall prediction information of UE orientation }}
$$

where $\mathcal{B}_{\mathbf{x}_{t-1}}\left(\boldsymbol{\alpha}_{t-1}\right)$ and $\mathcal{B}_{\mathbf{u}_{t-1}}\left(\boldsymbol{\alpha}_{t-1}\right)$ denote the associated CRLBs at the previous time slot $t-1$.

The initial CRLBs $\mathcal{B}_{\mathbf{x}_{0}}\left(\boldsymbol{\alpha}_{0}\right)$ and $\mathcal{B}_{\mathbf{u}_{0}}\left(\boldsymbol{\alpha}_{0}\right)$ are infinitely large; i.e., their FIMs $\mathcal{J}_{\mathbf{x}_{0}}\left(\boldsymbol{\alpha}_{0}\right)=\mathbf{0}$ and $\mathcal{J}_{\mathbf{u}_{0}}\left(\boldsymbol{\alpha}_{0}\right)=\mathbf{0}$, since there is no UE location parameter estimate at the initial stage. In this case, $\boldsymbol{\chi}_{\text {pred, }, 1}^{\circ}$ and $\mathbf{U}_{\text {pred, }, 1}^{\circ}$ at the first time slot reduce to $\chi_{\text {pred }, 1}^{\circ}=\chi_{\text {prior }}$ and $\mathbf{U}_{\text {pred }, 1}^{\circ}=\mathbf{U}_{\text {prior }}$, respectively.

2) Resolution Information: In (20) and (21), the resolution information $\mathcal{D}_{\mathbf{x}_{t}}\left(\mathbf{x}_{t}, \mathbf{u}_{t}\right)$ and $\mathcal{D}_{\mathbf{u}_{t}}\left(\mathbf{x}_{t}, \mathbf{u}_{t}\right)$ of UE location and orientation, respectively, are given by

$$
\begin{aligned}
& \mathcal{D}_{\mathbf{x}_{t}}\left(\mathbf{x}_{t}, \mathbf{u}_{t}\right)=\mathbf{H}\left(\mathbf{x}_{t}\right) \mathbf{K}\left(\mathbf{u}_{t}\right) \mathbf{F}\left(\mathbf{x}_{t}\right)\left(\mathbf{K}\left(\mathbf{u}_{t}\right)\right)^{\top}\left(\mathbf{H}\left(\mathbf{x}_{t}\right)\right)^{\top} \\
& \mathcal{D}_{\mathbf{u}_{t}}\left(\mathbf{x}_{t}, \mathbf{u}_{t}\right)=\mathcal{R}_{t}\left(\mathbf{G}\left(\mathbf{x}_{t}\right)\right)^{\top} \mathbf{V}\left(\mathbf{x}_{t}, \mathbf{u}_{t}\right) \mathbf{G}\left(\mathbf{x}_{t}\right) \mathcal{R}_{t}^{\top}
\end{aligned}
$$

where $\mathbf{H}\left(\mathbf{x}_{t}\right) \in \mathbb{R}^{3 \times 3\left|\Omega_{t}\right|}$ and $\mathbf{G}\left(\mathbf{x}_{t}\right) \in \mathbb{R}^{\left|\Omega_{t}\right| \times 3}$ are given, respectively, by

$$
\begin{aligned}
\mathbf{H}\left(\mathbf{x}_{t}\right)= & {\left[\rho_{m, t}^{-3} \mathbf{D}_{m}\left(\mathbf{x}_{t}\right) \mid \forall m \in \Omega_{t}\right], } \\
\mathbf{D}_{m}\left(\mathbf{x}_{t}\right)= & r(r+1)\left(\mathbf{e}_{m, t}^{\top} \mathbf{v}_{m}\right)^{r-1} \mathbf{v}_{m} \mathbf{e}_{m, t}^{\top} \\
& +(r+1)\left(\mathbf{e}_{m, t}^{\top} \mathbf{v}_{m}\right)^{r} \mathbf{I}_{3} \\
& -(r+3)(r+1)\left(\mathbf{e}_{m, t}^{\top} \mathbf{v}_{m}\right)^{r} \mathbf{e}_{m, t} \mathbf{e}_{m, t}^{\top}, \\
\mathbf{G}\left(\mathbf{x}_{t}\right)= & \operatorname{vec}\left[\mathbf{g}_{m}^{\top}\left(\mathbf{x}_{t}\right) \mid \forall m \in \Omega_{t}\right], \\
\mathbf{g}_{m}\left(\mathbf{x}_{t}\right)= & \frac{(r+1)\left(\left(\mathbf{x}_{t}-\mathbf{p}_{m}\right)^{\top} \mathbf{v}_{m}\right)^{r}}{\left\|\mathbf{x}_{t}-\mathbf{p}_{m}\right\|_{2}^{r+3}}\left(\mathbf{p}_{m}-\mathbf{x}_{t}\right),
\end{aligned}
$$

while $\mathbf{F}\left(\mathbf{x}_{t}\right)$ and $\mathbf{V}\left(\mathbf{x}_{t}, \mathbf{u}_{t}\right) \in \mathbb{S}^{\left|\Omega_{t}\right|}$ are given by (30) and (31), respectively. In addition, $\mathbf{K}\left(\mathbf{u}_{t}\right) \in \mathbb{R}^{3\left|\Omega_{t}\right| \times\left|\Omega_{t}\right|}=\mathbf{I}_{\left|\Omega_{t}\right|} \otimes$ $\frac{\mathbf{u}_{t}}{\left\|\mathbf{u}_{t}\right\|_{2}}$ with $\otimes$ being the Kronecker product and $\mathbf{I}_{3}$ being a 3-dimensional identity matrix, and $\mathcal{R}_{t}=\frac{\left\|\mathbf{u}_{t}\right\|_{2}^{2} \mathbf{I}_{3}-\mathbf{u}_{t} \mathbf{u}_{t}^{\top}}{\left\|\mathbf{u}_{t}\right\|_{2}^{3}}$.

\section{B. Remarks on VLP Error Bound}

In light of theorem 1, we draw the following remarks on the formation of the overall VLP error bound at each time slot.

Remark 1. (1) It is shown in (20) and (21) that the overall VLP information (i.e., inverse CRLB meaning the accuracy) 


$$
\begin{gathered}
\mathbf{F}\left(\mathbf{x}_{t}\right)=\mathbf{I}_{\left|\Omega_{t}\right|}-\mathbf{G}\left(\mathbf{x}_{t}\right) \boldsymbol{\mathcal { R }}_{t}^{\top} \underbrace{\left(\mathcal{R}_{t}\left(\mathbf{G}\left(\mathbf{x}_{t}\right)\right)^{\top} \mathbf{G}\left(\mathbf{x}_{t}\right) \boldsymbol{\mathcal { R }}_{t}^{\top}+\mathbf{U}_{\text {pred }, t}^{\circ}\right)^{-1}}_{\text {UE orientation uncertainty }} \mathcal{R}_{t}\left(\mathbf{G}\left(\mathbf{x}_{t}\right)\right)^{\top} \\
\mathbf{V}\left(\mathbf{x}_{t}, \mathbf{u}_{t}\right)=\mathbf{I}_{\left|\Omega_{t}\right|}-\left(\mathbf{K}\left(\mathbf{u}_{t}\right)\right)^{\top}\left(\mathbf{H}\left(\mathbf{x}_{t}\right)\right)^{\top} \underbrace{\left(\mathbf{H}\left(\mathbf{x}_{t}\right) \mathbf{K}\left(\mathbf{u}_{t}\right)\left(\mathbf{K}\left(\mathbf{u}_{t}\right)\right)^{\top}\left(\mathbf{H}\left(\mathbf{x}_{t}\right)\right)^{\top}+\chi_{\text {pred }, t}^{\circ}\right)^{-1}}_{\text {UE location uncertainty }} \mathbf{H}\left(\mathbf{x}_{t}\right) \mathbf{K}\left(\mathbf{u}_{t}\right)
\end{gathered}
$$

is the summation of observation information and movement prediction information. (11) In addition, it is shown in (22) and (23) that the overall prediction information (e.g., $\left.\chi_{\text {pred, } t}^{\circ}\right)$ at the current time slot $t$ is comprised of the prior knowledge (e.g., $\left.\chi_{\text {prior }}\right)$ and the prediction information (e.g., $\chi_{\text {pred, } t}$ ) via time recursion. The time-recursion information gain depends on the previous VLP error $\left(\boldsymbol{B}_{\mathbf{x}_{t-1}}\left(\boldsymbol{\alpha}_{t-1}\right)\right.$ and $\mathcal{B}_{\mathbf{u}_{t-1}}\left(\boldsymbol{\alpha}_{t-1}\right)$ ) and UE mobility (characterized by state transition precision matrices $\chi_{\text {trans }}^{-1}$ and $\mathbf{U}_{\text {trans }}^{-1}$ ). (111) Thus, the present-time VLP performance depends on the UE mobility and the previous VLP performance, in addition to observation information and the prior knowledge from inertial readings. Hence, the essence of time-domain localization cooperation in mobile UE tracking is the propagation of the associated localization information, in an information-theoretic view.

This information formation process implies a phenomenon of VLP error evolution from $\mathcal{B}_{\mathbf{x}_{t-1}}\left(\boldsymbol{\alpha}_{t-1}\right)$ to $\mathcal{B}_{\mathbf{x}_{t}}\left(\boldsymbol{\alpha}_{t}\right)$ (and also $\mathcal{B}_{\mathbf{u}_{t}}\left(\boldsymbol{\alpha}_{t}\right)$ ) in time-domain, which will affect the long-term VLP performance and will be analyzed in Section IV.

Remark 2. It is shown in (20)-(29) that the VLP performance is affected by (1) the number of independent measurement sources, i.e., $\left|\Omega_{t}\right|$, (11) LED deployment $\left\{\mathbf{p}_{m} \mid \forall m \in \Omega_{t}\right\}$, (111) LED orientations $\left\{\mathbf{v}_{m} \mid \forall m \in \Omega_{t}\right\}$, (1v) measurement resolution $\left\{\mathcal{D}_{\mathbf{x}_{t}}\left(\boldsymbol{\alpha}_{t}\right), \mathcal{D}_{\mathbf{u}_{t}}\left(\boldsymbol{\alpha}_{t}\right) \mid \forall m \in \Omega_{t}\right\}$, (v) prediction knowledge of UE location parameters and (vi) SNR.

In addition, on the meaning of resolution information matrix, we have the following remark.

Remark 3. It is shown in (20) and (21) that the observation information is determined by the resolution information of VLP systems and the noise strength (equivalently SNR). The resolution information $\mathcal{D}_{\mathbf{x}_{t}}\left(\mathbf{x}_{t}, \mathbf{u}_{t}\right)$ and $\mathcal{D}_{\mathbf{u}_{t}}\left(\mathbf{x}_{t}, \mathbf{u}_{t}\right)$ depends on the choice of measurement signals and the VLP system geometry, which is essentially affected by its measurement function $h_{m}\left(\mathbf{x}_{t}, \mathbf{u}_{t}\right)$. This metric indicates the capability to recognize the difference in $\mathbf{x}_{t}$ or $\mathbf{u}_{t}$, for a given variation of measurement $\mathbf{z}_{t}$ [18], [20]. A zero-valued resolution information matrix means the unobservability of a parameter, since the measurement will remain invariant for different values of this parameter. Obviously, the measurement system with a high resolution (sensitivity) leads to a good VLP performance.

The above CRLB reveals the VLP performance at each time slot, which can serve as a performance benchmark for practical VLP systems. However, the long-term VLP performance as time flies is still not understood.

\section{EVOLUTION OF VLP ERROR}

In this section, we analyse the time-domain error evolution (TDEE) to reveal the long-term VLP performance.

\section{A. Evolution Function of VLP Error}

It is implied in theorem 1 and remark 1 that there will be time-domain VLP error evolution in the UE location tracking process. Specifically, the current VLP error performance $\mathcal{B}_{\mathbf{x}_{t}}$ and $\mathcal{B}_{\mathbf{u}_{t}}{ }^{3}$ depend on their previous-time-slot values $\mathcal{B}_{\mathbf{x}_{t-1}}$ and $\mathcal{B}_{\mathbf{u}_{t-1}}$, respectively. Hence, $\mathcal{B}_{\mathbf{x}_{t}}$ can be viewed as a function of $\mathcal{B}_{\mathbf{x}_{t-1}}$, named the error evolution function. The associated VLP error evolution functions $\left(\mathcal{B}_{\mathbf{x}_{t-1}} \mapsto \mathcal{B}_{\mathbf{x}_{t}}\right.$ and $\mathcal{B}_{\mathbf{u}_{t-1}} \mapsto$ $\mathcal{B}_{\mathbf{u}_{t}}$ ) for UE location and orientation have been potentially characterized in (20) and (21), respectively, and we restate them in the following for the purpose of clarity,

$$
\begin{aligned}
& \mathcal{B}_{\mathbf{x}_{t}}=\left(\mathcal{H}_{\mathbf{x}_{t}}^{\text {obs }}+\boldsymbol{\chi}_{\text {prior }}+\left(\boldsymbol{\chi}_{\text {trans }}^{-1}+\mathcal{B}_{\mathbf{x}_{t-1}}\right)^{-1}\right)^{-1} \\
& \mathcal{B}_{\mathbf{u}_{t}}=\left(\mathcal{H}_{\mathbf{u}_{t}}^{\text {obs }}+\mathbf{U}_{\text {prior }}+\left(\mathbf{U}_{\text {trans }}^{-1}+\mathcal{B}_{\mathbf{u}_{t-1}}\right)^{-1}\right)^{-1}
\end{aligned}
$$

where $\mathcal{H}_{\mathbf{x}_{t}}^{\text {obs }}, \chi_{\text {prior }}$ and $\chi_{\text {trans }}$ can be viewed as constants for the evolution of $\mathcal{B}_{\mathbf{x}_{t}}$, and so can $\mathcal{H}_{\mathbf{u}_{t}}^{\text {obs }}, \mathbf{U}_{\text {prior }}$ and $\mathbf{U}_{\text {trans. }}$

\section{B. Assumptions on VLP System}

We can see from (32) and (33) that the time-domain error evolution is essentially a fixed-point iteration over matrix, and hence its convergence is fully determined by the contractionmap property of the above evolution functions.

To establish its convergence, we first give the following assumptions on the VLP measurement dynamics:

- (A1) $\mathcal{H}_{\mathbf{x}_{t}}^{\text {obs }} \rightarrow \mathcal{H}_{\mathbf{x} \star}^{\text {obs }}$, as $t \rightarrow \infty$,

- (A2) $\mathcal{H}_{\mathbf{u}_{t}}^{\text {obs }} \rightarrow \mathcal{H}_{\mathbf{u} \star}^{\text {obs }}$, as $t \rightarrow \infty$,

where $\mathcal{H}_{\mathbf{x} \star}^{\text {obs }}$ and $\mathcal{H}_{\mathbf{u} \star}^{\text {obs }}$ are in $\mathbb{S}_{+}^{3}$, and it should be noted that $\mathcal{H}_{\mathbf{x}_{t}}^{\text {obs }}$ and $\mathcal{H}_{\mathbf{u}_{t}}^{\text {obs }}$ have been given by (20) and (21), respectively.

Assumptions A1 and A2 mean that the observation information w.r.t. UE location and orientation should converge over time. In practice, the observation information $\mathcal{H}_{\mathbf{x}_{t}}^{\text {obs }}$ and $\mathcal{H}_{\mathbf{u}_{t}}^{\text {obs }}$ are more likely to vary within a bounded area due to the UE mobility. Yet, we still impose those assumptions on the long-term observation information to facilitate the convergence analysis and then gain insights into the long-term VLP performance, and these ideal assumptions will not affect the obtained insights.

It should be noted that the observation information $\mathcal{H}_{\mathbf{x}_{t}}^{\text {obs }}$ and hence its limit $\mathcal{H}_{\mathrm{x} \star}^{\text {obs }}$ are determined by the measurement resolution (also the VLP system geometry and the number of LED sources) and SNR, as shown in (20).

\section{Convergence Analysis of TDEE}

We give the following theorems 2 and 3 to formally establish the convergence and the stable state of TDEE, respectively.

\footnotetext{
${ }^{3}$ We have dropped the notation $\left(\mathbf{x}_{t}, \mathbf{u}_{t}\right)$ for clarity.
} 
For ease of notation, let $\mathcal{B}_{\mathrm{x}}^{\star}$ and $\mathcal{B}_{\mathrm{u}}^{\star}$ be the limit point (stable state) of $\mathcal{B}_{\mathbf{x}_{t}}$ and $\mathcal{B}_{\mathbf{u}_{t}}$, respectively. In addition, let $\mathcal{H}_{\mathbf{x} \star}^{\sharp}$ and $\mathcal{H}_{\mathbf{u} \star}^{\sharp}$ be the limit points of equivalent observation information matrices, given by

$$
\begin{aligned}
& \mathcal{H}_{\mathbf{x} \star}^{\sharp}=\mathcal{H}_{\mathbf{x} \star}^{\text {obs }}+\chi_{\text {prior }}, \\
& \mathcal{H}_{\mathbf{u} \star}^{\sharp}=\mathcal{H}_{\mathbf{u} \star}^{\text {obs }}+\mathbf{U}_{\text {prior }} .
\end{aligned}
$$

Theorem 2 (Convergence of TDEE): If $\mathrm{A} 1$ and $\mathrm{A} 2$ are satisfied, UE location and orientation error bounds $\mathcal{B}_{\mathbf{x}_{t}}$ and $\mathcal{B}_{\mathbf{u}_{t}}$ must converge to two certain fixed points $\mathcal{B}_{\mathbf{x}}^{\star}$ and $\mathcal{B}_{\mathbf{u}}^{\star}$ in $\mathbb{S}^{3}$, respectively, at linear convergence rates, as $t \rightarrow \infty$, i.e., ${ }^{4}$

$$
\begin{aligned}
& \lim _{t \rightarrow \infty} \frac{\left\|\mathcal{B}_{\mathbf{x}_{t+1}}-\mathcal{B}_{\mathbf{x}_{t}}\right\|_{F}}{\left\|\mathcal{B}_{\mathbf{x}_{t}}-\mathcal{B}_{\mathbf{x}_{t-1}}\right\|_{F}}=1-\kappa_{\mathbf{x}} \in(0,1), \\
& \lim _{t \rightarrow \infty} \frac{\left\|\mathcal{B}_{\mathbf{u}_{t+1}}-\mathcal{B}_{\mathbf{u}_{t}}\right\|_{F}}{\left\|\mathcal{B}_{\mathbf{u}_{t}}-\mathcal{B}_{\mathbf{u}_{t-1}}\right\|_{F}}=1-\kappa_{\mathbf{u}} \in(0,1),
\end{aligned}
$$

where $\kappa_{\mathbf{x}}$ and $\kappa_{\mathbf{u}}$ are two constants in $(0,1)$, given by (38) and (39), respectively, in which the closed-form expressions of $\mathcal{B}_{\mathrm{x}}^{\star}$ and $\mathcal{B}_{\mathrm{u}}^{\star}$ will be determined in the following theorem 3 .

Proof: See the proof in APPENDIX B.

This means that the VLP error tends to remain invariant at its limiting point over time. This limiting point is named the stable state of VLP error bound (long-term VLP performance), which is actually the fixed-point solution (established later) of the associated TDEE function. In practice, for time-varying $\mathcal{H}_{\mathbf{x}_{t}}^{\text {obs }}$ and $\mathcal{H}_{\mathbf{u}_{t}}^{\text {obs }}$ within a bounded region, the limiting point of CRLB iteration is likely to be varying within a bounded region too, since the following theorem will establish that the closed-form expression of TDEE limiting point is a Lipschizcontinuous function of observation information.

Theorem 3 (Closed-Form Stable State Of TDEE): If A1 and $\mathrm{A} 2$ are satisfied, the closed-form expressions of the stable states (limit points) $\mathcal{B}_{\mathbf{x}}^{\star}$ and $\mathcal{B}_{\mathbf{u}}^{\star}$ of $\mathcal{B}_{\mathbf{x}_{t}}$ and $\mathcal{B}_{\mathbf{u}_{t}}$, respectively, as $t \rightarrow \infty$, are given by

$$
\begin{aligned}
\mathcal{B}_{\mathbf{x}}^{\star} & =\frac{1}{2} \boldsymbol{\chi}_{\text {trans }}^{-\frac{1}{2}}\left(\mathbf{I}_{3}+4 \boldsymbol{\chi}_{\text {trans }}^{\frac{1}{2}}\left(\mathcal{H}_{\mathbf{x} \star}^{\sharp}\right)^{-1} \boldsymbol{\chi}_{\text {trans }}^{\frac{1}{2}}\right)^{\frac{1}{2}} \boldsymbol{\chi}_{\text {trans }}^{-\frac{1}{2}}-\frac{1}{2} \boldsymbol{\chi}_{\text {trans }}^{-1} \\
\mathcal{B}_{\mathbf{u}}^{\star} & =\frac{1}{2} \mathbf{U}_{\text {trans }}^{-\frac{1}{2}}\left(\mathbf{I}_{3}+4 \mathbf{U}_{\text {trans }}^{\frac{1}{2}}\left(\mathcal{H}_{\mathbf{u} \star}^{\sharp}\right)^{-1} \mathbf{U}_{\text {trans }}\right)^{\frac{1}{2}} \mathbf{U}_{\text {trans }}^{-\frac{1}{2}}-\frac{1}{2} \mathbf{U}_{\text {trans }}^{-1}
\end{aligned}
$$

where $\mathcal{H}_{\mathbf{x} \star}^{\sharp}$ and $\mathcal{H}_{\mathbf{u} \star}^{\sharp}$ are given by (34) and (35), respectively.

Proof: See the proof in APPENDIX C.

Remark 4 . The above stable state quantifies the long-term VLP error performance, which is totally determined by the observation information $\left(\mathcal{H}_{\mathbf{x} \star}^{\text {obs }}\right.$ and $\mathcal{H}_{\mathbf{u} \star}^{\text {obs }}$ ), prior information $\left(\chi_{\text {prior }}\right.$ and $\left.\mathbf{U}_{\text {prior }}\right)$ and UE movement information ( $\chi_{\text {trans }}$ and $\left.\mathbf{U}_{\text {trans }}\right)$. Since $\mathcal{B}_{\mathrm{x}}^{\star}$ is a Lipschiz-continuous function of the observation information $\mathcal{H}_{\mathbf{x}}^{\text {obs }}$ (as shown in theorem 3) and all information matrices are full-rank in practice, the stable state $\mathcal{B}_{\mathbf{x}}^{\star}$ must be bounded due to the bounded $\mathcal{H}_{\mathbf{x}_{t}}^{\text {obs }}$, and so must be $\mathcal{B}_{\mathbf{u}}^{\star}$. This means, we can drop assumptions A1 and A2 on measurement dynamics, and the mobile UE tracking system can achieve a bounded long-term performance in practice.

In practice, there is usually less information of the UE location parameters at the initial time (i.e., large initial error

\footnotetext{
${ }^{4}$ It is equivalent that $\lim _{t \rightarrow \infty} \frac{\left\|\mathcal{B}_{\mathbf{x}_{t+1}}-\mathcal{B}_{\mathbf{\times}}^{\star}\right\|_{F}}{\left\|\mathcal{B}_{\mathbf{x}_{t}}-\mathcal{B}_{\mathbf{\times}}^{\star}\right\|_{F}}=1-\kappa_{\mathbf{x}}$, and so is $\mathcal{B}_{\mathbf{u}_{t}}$.
}

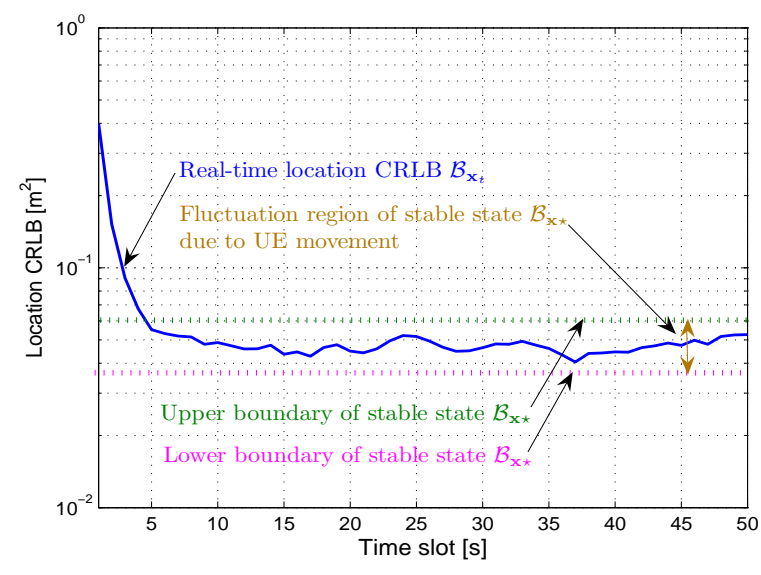

Fig. 2. An illustration of the fluctuation of VLP error stable state due to UE movement (time-varying observation information).

bounds $\mathcal{B}_{\mathbf{x}_{t}}$ and $\mathcal{B}_{\mathbf{u}_{t}}$ ). The following theorem establishes that the VLP errors are monotonically non-increasing over time.

Theorem 4 (Monotonically Non-Increasing Of TDEE): If the observation information matrices $\mathcal{H}_{\mathbf{x}_{t}}^{\text {obs }}$ and $\mathcal{H}_{\mathbf{u}_{t}}^{\text {obs }}$ are nondecreasing over time and bounded from above by their limit states $\mathcal{H}_{\mathbf{x} \star}^{\text {obs }}$ and $\mathcal{H}_{\mathbf{u} \star}^{\text {obs }}$, respectively, i.e.,

- (B1) $\mathcal{H}_{\mathbf{x}_{t-1}}^{\text {obs }} \preceq \mathcal{H}_{\mathbf{x}_{t}}^{\text {obs }} \preceq \mathcal{H}_{\mathrm{x} \star}^{\text {obs }}, \forall t>0$,

- (B2) $\mathcal{H}_{\mathbf{u}_{t-1}}^{\text {obs }} \preceq \mathcal{H}_{\mathbf{x}_{t}}^{\text {obs }} \preceq \mathcal{H}_{\mathbf{u} \star}^{\text {obs }}, \forall t>0$,

then the VLP error bounds $\mathcal{B}_{\mathbf{x}_{t}}$ and $\mathcal{B}_{\mathbf{u}_{t}}$ are both monotonically non-increasing over time, i.e.,

$$
\begin{aligned}
& \mathcal{B}_{\mathbf{x}_{t}} \preceq \mathcal{B}_{\mathbf{x}_{t-1}}, \quad \forall t>0 \\
& \mathcal{B}_{\mathbf{u}_{t}} \preceq \mathcal{B}_{\mathbf{u}_{t-1}}, \quad \forall t>0 .
\end{aligned}
$$

Proof: See proof in APPENDIX D.

Two stronger conditions $\mathrm{B} 1$ and $\mathrm{B} 2$ than $\mathrm{A} 1$ and $\mathrm{A} 2$, respectively, are assumed in theorem 4 to exactly establish the non-increasing VLP error bounds, which requires that the observation information is non-decreasing over time.

Although B1 and B2 are not necessarily satisfied in practice, they still provide a useful indication of practical TDEE properties. In practice, the observation information may vary over time within a compact region, e.g., $\mathcal{H}_{\mathbf{x} t}^{\text {obs }} \in\left[\mathcal{H}_{\mathbf{x}, \min }^{\text {obs }}, \mathcal{H}_{\mathbf{x}, \max }^{\text {obs }}\right]$ and $\mathcal{H}_{\mathbf{u}_{t}}^{\text {obs }} \in\left[\mathcal{H}_{\mathbf{u}, \text { min }}^{\text {obs }}, \mathcal{H}_{\mathbf{u}, \text { max }}^{\text {obs }}\right]$, due to the dynamics of UE location and orientation. In such a common case, the VLP error bounds will gradually decrease and eventually fluctuate within a compact region, i.e., $\mathcal{B}_{\mathbf{x}_{t}} \in\left[\mathcal{B}_{\mathbf{x}, \text { min }}, \mathcal{B}_{\mathbf{x}, \text { max }}\right]$ and $\mathcal{B}_{\mathbf{u}_{t}} \in\left[\mathcal{B}_{\mathbf{u}, \text { min }}, \mathcal{B}_{\mathbf{u}, \text { max }}\right]$, for $t \geq t_{\text {stable }}$ with some $t_{\text {stable }}>0$, as illustrated in Fig. 2, where $\mathcal{B}_{\mathbf{x}, \text { min }}$ relates to $\mathcal{H}_{\mathbf{x}, \text { max }}^{\text {obs }}$, while $\mathcal{B}_{\mathbf{x}, \text { max }}$ relates to $\mathcal{H}_{\mathbf{x}, \text { min }}^{\text {obs }}$ (see stable state expressions in theorem 3), and the same applies to $\mathcal{B}_{\mathbf{u} \text {, min }}$ and $\mathcal{B}_{\mathbf{u} \text {, max }}$. This trend has been verified by numerical simulations.

In a summary, we draw the following remark on VLP error convergence behavior from theorems 2-4.

Remark 5. VLP errors will converge downwards to their stable states under non-decreasing assumptions of observation information. For the common case that the observation information varies within a bounded region, the VLP errors will gradually decrease and then fluctuate within a bounded region, and the fluctuation region of the VLP errors depends on the range of the observation information variation. 


$$
\begin{aligned}
& \kappa_{\mathbf{x}}=\left\|\left(\chi_{\text {trans }}^{-1}+\mathcal{B}_{\mathbf{x}}^{\star}\right)\left(\chi_{\text {trans }}^{-1}+\mathcal{B}_{\mathbf{x}}^{\star}+\left(\mathcal{H}_{\mathbf{x} \star}^{\sharp}\right)^{-1}\right)^{-2}\left(\chi_{\text {trans }}^{-1}+\mathcal{B}_{\mathbf{x}}^{\star}+2\left(\mathcal{H}_{\mathbf{x} \star}^{\sharp}\right)^{-1}\right)\right\|_{F}, \\
& \kappa_{\mathbf{u}}=\left\|\left(\chi_{\text {trans }}^{-1}+\mathcal{B}_{\mathbf{u}}^{\star}\right)\left(\boldsymbol{\chi}_{\text {trans }}^{-1}+\mathcal{B}_{\mathbf{u}}^{\star}+\left(\mathcal{H}_{\mathbf{u} \star}^{\sharp}\right)^{-1}\right)^{-2}\left(\boldsymbol{\chi}_{\text {trans }}^{-1}+\mathcal{B}_{\mathbf{u}}^{\star}+2\left(\mathcal{H}_{\mathbf{u} \star}^{\sharp}\right)^{-1}\right)\right\|_{F} .
\end{aligned}
$$

The above analysis theoretically establishes the downwards convergence of the TDEE process. However, the associated physical essence is still not understood. In fact, the downwards convergence behavior of TEDD is resulted from the battle between the observation information and the UE mobilitycaused information loss, as elaborated in the following.

\section{The EFFect Of UE Mobility}

In this section, we shall analysis the effect of UE mobility on the VLP performance to reveal the physical essence behind the TDEE convergence behavior and explicate what happens during this TDEE process.

\section{A. UE Mobility-Caused Information Loss}

Due to UE mobility (characterized by $\chi_{\text {trans }}$ and $\mathbf{U}_{\text {trans }}$ ), the prediction information $\chi_{\text {pred, } t}^{\circ}$ and $\mathbf{U}_{\text {pred, } t}^{\circ}$ will be less than the previous posterior information $\mathcal{J}_{\mathbf{x}_{t-1}}\left(\boldsymbol{\alpha}_{t-1}\right)$ and $\mathcal{J}_{\mathbf{u}_{t-1}}\left(\boldsymbol{\alpha}_{t-1}\right)$ (see (20) and (21)), respectively. In other words, the UE mobility will cause a reduction in the VLP information, as established below. Let $\boldsymbol{\Upsilon}_{\mathbf{x}_{t}}$ and $\boldsymbol{\Upsilon}_{\mathbf{u}_{t}} \in \mathbb{S}^{3}$ denote the UE mobility-caused information loss of UE location and orientation, respectively.

Corollary 1 (Non-Negative Information Loss): At each time slot, there will be an non-negative information loss $\Upsilon_{\mathbf{x}_{t}} \succeq \mathbf{0}$ and $\boldsymbol{\Upsilon}_{\mathbf{u}_{t}} \succeq \mathbf{0}$ in the UE location and orientation estimates, respectively, due to UE mobility, which are given by

$$
\begin{aligned}
& \boldsymbol{\Upsilon}_{\mathbf{x}_{t}}=\mathcal{J}_{\mathbf{x}_{t-1}}\left(\boldsymbol{\alpha}_{t-1}\right)-\left(\chi_{\text {trans }}^{-1}+\mathcal{J}_{\mathbf{x}_{t-1}}^{-1}\left(\boldsymbol{\alpha}_{t-1}\right)\right)^{-1} \\
& \boldsymbol{\Upsilon}_{\mathbf{u}_{t}}=\mathcal{J}_{\mathbf{u}_{t-1}}\left(\boldsymbol{\alpha}_{t-1}\right)-\left(\mathbf{U}_{\text {trans }}^{-1}+\mathcal{J}_{\mathbf{u}_{t-1}}^{-1}\left(\boldsymbol{\alpha}_{t-1}\right)\right)^{-1}
\end{aligned}
$$

Proof: (42) and (43) can be easily obtained as per (22) and (23), respectively. In addition, since $\chi_{\text {trans }}$ and $\mathbf{U}_{\text {trans }} \succeq \mathbf{0}$, we can conclude that $\boldsymbol{\Upsilon}_{\mathbf{x}_{t}}$ and $\boldsymbol{\Upsilon}_{\mathbf{u}_{t}} \succeq \mathbf{0}$.

This means, the prediction information must be not larger than the previous-time-slot VLP information, which will lead to the reduction of the overall VLP performance. Specially, combining (42) and (43) with theorem 1, the VLP error bounds of UE location and orientation can be cast as the following forms, respectively, ${ }^{5}$

$$
\begin{gathered}
\mathcal{B}_{\mathbf{x}_{t}}\left(\mathbf{x}_{t}, \mathbf{u}_{t}\right)=(\underbrace{\underbrace{\mathcal{H}_{\mathbf{x}_{t}}^{\text {obs }}\left(\mathbf{x}_{t}, \mathbf{u}_{t}\right)}_{\begin{array}{c}
\text { Total location information } \mathcal{J}_{\mathbf{x}_{t}}\left(\mathbf{x}_{t}, \mathbf{u}_{t}\right) \\
\text { information } \boldsymbol{\chi}_{\text {pred }, t}^{\circ}
\end{array}}+\underbrace{\mathcal{J}_{\mathbf{x}_{t-1}}-\mathbf{\Upsilon}_{\mathbf{x}_{t}}})^{-1},}_{\begin{array}{c}
\text { Overall observation } \\
\text { information }
\end{array}} \\
\mathcal{B}_{\mathbf{u}_{t}}\left(\mathbf{x}_{t}, \mathbf{u}_{t}\right)= \\
\underbrace{\underbrace{\mathcal{H}_{\mathbf{u}_{t}}^{\text {obs }}\left(\mathbf{x}_{t}, \mathbf{u}_{t}\right)}_{\text {Total location information } \mathcal{J}_{\mathbf{u}_{t}\left(\mathbf{x}_{t}, \mathbf{u}_{t}\right)}}}_{\begin{array}{c}
\text { Overall observation } \\
\text { information }
\end{array}}+\underbrace{\mathcal{J}_{\mathbf{u}_{t-1}}-\mathbf{\Upsilon}_{\mathbf{u}_{t}}}_{\begin{array}{c}
\text { Overall prediction } \\
\text { information } \boldsymbol{\chi}_{\text {pred }, t}^{\circ}
\end{array}})^{-1},
\end{gathered}
$$

\footnotetext{
${ }^{5}$ We have dropped $\left(\mathbf{x}_{t}, \mathbf{u}_{t}\right)$ in information notations for brevity.
}

in which the UE mobility-caused information loss and the overall VLP performance reduction are explicitly shown.

\section{B. Increasing of Information Loss}

It is established in theorem 4 that the VLP error will decrease over time, i.e., $\mathcal{B}_{\mathbf{x}_{t}}\left(\boldsymbol{\alpha}_{t}\right) \preceq \mathcal{B}_{\mathbf{x}_{t-1}}\left(\boldsymbol{\alpha}_{t-1}\right)$. The following corollary shows that the UE mobility-caused information loss will increase with the decreasing of VLP errors.

Corollary 2 (Increasing of UE Mobility-Caused Information Loss): For the decreasing VLP errors $\mathcal{B}_{\mathbf{x}_{t}}\left(\boldsymbol{\alpha}_{t}\right)$ and $\mathcal{B}_{\mathbf{u}_{t}}\left(\boldsymbol{\alpha}_{t}\right)$ over time, the prediction information loss $\boldsymbol{\Upsilon}_{\mathbf{x}_{t}}$ and $\boldsymbol{\Upsilon}_{\mathbf{u}_{t}}$ follows the following inequalities,

$$
\begin{aligned}
\boldsymbol{\Upsilon}_{\mathbf{x}_{t}} \succeq \boldsymbol{\Upsilon}_{\mathbf{x}_{t-1}}, \text { given } \mathcal{B}_{\mathbf{x}_{t}}\left(\boldsymbol{\alpha}_{t}\right) \preceq \mathcal{B}_{\mathbf{x}_{t-1}}\left(\boldsymbol{\alpha}_{t-1}\right), \\
\boldsymbol{\Upsilon}_{\mathbf{u}_{t}} \succeq \boldsymbol{\Upsilon}_{\mathbf{u}_{t-1}}, \text { given } \mathcal{B}_{\mathbf{u}_{t}}\left(\boldsymbol{\alpha}_{t}\right) \preceq \mathcal{B}_{\mathbf{u}_{t-1}}\left(\boldsymbol{\alpha}_{t-1}\right)
\end{aligned}
$$

Proof: This can be verified as per (42) and (43).

Remark 6. The more accurate VLP result will cause the larger information reduction $\Upsilon_{\mathbf{x}_{t}}$ and $\boldsymbol{\Upsilon}_{\mathbf{u}_{t}}$, due to UE mobilities. This means, as the VLP accuracies $\mathcal{J}_{\mathbf{x}_{t-1}}\left(\boldsymbol{\alpha}_{t-1}\right)$ and $\mathcal{J}_{\mathbf{u}_{t-1}}\left(\boldsymbol{\alpha}_{t-1}\right)$ increase over time, the information loss $\boldsymbol{\Upsilon}_{\mathbf{x}_{t}}$ and $\Upsilon_{\mathbf{u}_{t}}$ will become larger and larger. Therefore, when VLP error reduces over time, the UE mobility-caused information loss will gradually increase unless the information loss is comparable to the observation information gain so that VLP error becomes invariant.

This VLP error evolution process is similar to the accelerating movement of a near-light-speed object. Specifically, for a near-light-speed object, the increased speed leads to the increased mass; Hence, given a fixed energy input, the speed increment will be gradually decreased due to the increasing mass. Therefore, this object will achieve an invariant speed approaching the light speed, for sustained energy input. Likewise, the increased VLP information $\mathcal{J}_{\mathbf{x}_{t-1}}\left(\boldsymbol{\alpha}_{t-1}\right)$ leads to increased information loss $\boldsymbol{\Upsilon}_{\mathbf{x}_{t}}$. Hence, given a fixed observation information gain $\mathcal{H}_{\mathbf{x}_{t}}^{\text {obs }}$, the prediction information gain $\chi_{\text {pred, } t}^{\circ}$ will be gradually reduced due to the increasing VLP information. Therefore, the VLP process will achieve a stable information approaching its limit point (see theorem 3), for a sustained information gain from observations.

\section{Physical Insights of TDEE}

The VLP error bounds $\mathcal{B}_{\mathbf{x}_{t}}\left(\mathbf{x}_{t}, \mathbf{u}_{t}\right)$ and $\mathcal{B}_{\mathbf{u}_{t}}\left(\mathbf{x}_{t}, \mathbf{u}_{t}\right)$ will gradually decrease over time, since the overall observation information $\mathcal{H}_{\mathbf{x}_{t}}^{\text {obs }}$ and $\mathcal{H}_{\mathbf{u}_{t}}^{\text {obs }}$ can compensate (be larger than) for the UE mobility-caused information loss $\boldsymbol{\Upsilon}_{\mathbf{x}_{t}}$ and $\boldsymbol{\Upsilon}_{\mathbf{x}_{t}}$, respectively. Yet, with the decrease of VLP error, the information loss will gradually increase as revealed in remark 6 . Thus, the VLP error reduction will become gradually slower such that it eventually achieves stability when the UE mobility-caused information loss is comparable to the observation information 
gain [19]. In other words, the VLP error will achieve a stable state, due to the balance between observation information gain and UE mobility-caused information loss.

The obtained closed-form CRLBs on UE location and orientation errors can not only serve as a performance benchmark for practical VLP systems, but also give insights into how system parameters and LED deployment affect the UE location and orientation estimate performance.

\section{Discussion: GUIDELINES FOR VeHICULAR VLP SySTEM OPTIMIZATION DESIGN}

In this section, based on the obtained CRLBs, we will provide guidelines for vehicular VLP system optimization, which will fundamentally improve the overall VLP performance.

\section{A. Power Allocation of LEDs}

Firstly, the obtained CRLB can provide insights into how to perform the best power allocation among various LEDs to achieve an efficient VLP system. Let $\varrho_{m}$ be SNR of the $m$ th LED, which is proportional to the LED emitting power, and let $\varrho_{t}=\operatorname{vec}\left[\varrho_{m} \mid \forall m \in \Omega_{t}\right]$ be the power allocation vector. Then, recalling (20), the real-time CRLB can be rewritten as ${ }^{6}$

$$
\mathcal{B}_{\mathbf{x}_{t}}\left(\varrho_{t} ; \boldsymbol{\alpha}_{t}\right)=\left(\sum_{m \in \Omega_{t}} \varrho_{m} \mathcal{D}_{\mathbf{x}_{t} ; m}\left(\boldsymbol{\alpha}_{t}\right)+\chi_{\text {pred }, t}\right)^{-1}
$$

which can be viewed as a function of $\varrho_{t}$, and $\mathcal{D}_{\mathbf{x}_{t} ; m}$ is the resolution matrix associated with $z_{m, t}$ which can be regarded as a parameter depending on UE location parameters $\boldsymbol{\alpha}_{t}$.

To achieve the lowest VLP error with a limited energy budget, we can perform a real-time LED power allocation via minimizing the the VLP error CRLB. However, the CRLB depends on the UE location parameters $\boldsymbol{\alpha}_{t}$, which are unknown beforehand. To get around this problem, conventional power allocation methods [25], [26] usually resort to a robust solution which is formulated as

$$
\begin{gathered}
\hat{\boldsymbol{\varrho}}_{t}=\arg \min _{\boldsymbol{\varrho}_{t}} \max _{\boldsymbol{\alpha}_{t} \in \mathbb{A}_{t}} \operatorname{trace}\left(\boldsymbol{\mathcal { B }}_{\mathbf{x}_{t}}\left(\boldsymbol{\varrho}_{t} ; \boldsymbol{\alpha}_{t}\right)\right) \\
\text { s.t. }\left\|\boldsymbol{\varrho}_{t}\right\|_{1} \leq \mathrm{SNR}_{\text {total }},
\end{gathered}
$$

where $S N R_{\text {total }}$ denotes the constant corresponding to the total emitting power of LEDs, and $\mathbb{A}_{t}$ denotes the uncertainty region of $\boldsymbol{\alpha}_{t}$. We can see that this is a non-convex problem due to the optimization of UE location parameters, which usually has a high computational cost, and thus an efficient optimization algorithm is needed [53]. In addition, the above robust solution ignores the UE location prior knowledge, and it is overconservative when the UE location uncertainty set is large, which degrades the system optimization performance.

Unlike the above conventional robust solution, we give an alternative solution to simplify the optimization problem, i.e.,

$$
\begin{aligned}
\hat{\varrho}_{t}= & \arg \min _{\boldsymbol{\varrho}_{t}} \int_{\boldsymbol{\alpha}_{t} \in \mathbb{A}_{t}} p\left(\boldsymbol{\alpha}_{t}\right) \operatorname{trace}\left(\mathcal{B}_{\mathbf{x}_{t}}\left(\varrho_{t} ; \boldsymbol{\alpha}_{t}\right)\right) \mathrm{d} \boldsymbol{\alpha}_{t}, \\
& \text { s.t. }\left\|\varrho_{t}\right\|_{1} \leq \mathrm{SNR}_{\text {total }},
\end{aligned}
$$

\footnotetext{
${ }^{6} \mathcal{B}_{\mathbf{x}_{t}}$ and $\mathcal{B}_{\mathbf{u}_{t}}$ have the same structure as above, and hence the associated insights will be applicable to them.
}

where $p\left(\boldsymbol{\alpha}_{t}\right)$ is the (Gaussian) priori function of $\boldsymbol{\alpha}_{t}$.

This problem is convex w.r.t. $\boldsymbol{\alpha}_{t}$; however, the cost function has no closed-form expression due to the complex integration. The optimization of cost function integration can be efficiently solved via resorting to stochastic optimization methods [54], [55], in which the complex integration can be approximated by iterations of the cost surrogate functions, and at each iteration the resulted subproblem (the optimization of $\varrho_{t}$ conditioned on a simple cost surrogate function) is expected to be convex and hence the computational cost is reduced.

\section{B. Let LED Beams Trace the Vehicular UE}

Secondly, it is possible to steer the orientation directions of LED sources to improve the VLP performance, since the obtained CRLB has explicitly shown its dependency on the LED orientation vector $\left\{\mathbf{v}_{m} \mid \forall m \in \Omega_{t}\right\}$, as revealed in theorem 1. Let $\boldsymbol{v}_{t}=\operatorname{vec}\left[\mathbf{v}_{m} \mid \forall m \in \Omega_{t}\right]$ be the collection of LED orientation vectors. Then, the real-time LED orientation steering can be achieved by solving the following problem,

$$
\begin{gathered}
\hat{\boldsymbol{v}}_{t}=\arg \min _{\boldsymbol{v}_{t}} \int_{\boldsymbol{\alpha}_{t} \in \mathbb{A}_{t}} p\left(\boldsymbol{\alpha}_{t}\right) \operatorname{trace}\left(\mathcal{B}_{\mathbf{x}_{t}}\left(\boldsymbol{v}_{t} ; \boldsymbol{\alpha}_{t}\right)\right) \mathrm{d} \boldsymbol{\alpha}_{t}, \\
\text { s.t. }\left\|\mathbf{v}_{m}\right\|_{2}=1, \forall m \in \Omega_{t},
\end{gathered}
$$

where the UE location CRLB $\mathcal{B}_{\mathbf{x}_{t}}\left(\boldsymbol{v}_{t}\right)$ is viewed as a function of $\boldsymbol{v}_{t}$, which has been given by (20). However, it is a nonconvex optimization problem w.r.t. the LED orientation vector $\boldsymbol{v}_{t}$, and hence an efficient optimization algorithm is needed.

\section{Smart Selection of LED Sources}

Thirdly, in practice, both VLP energy consumption and VLP performance are related to the active LED set. Hence, it is desired to conduct a smart LED source selection procedure to achieve an accurate VLP solution with economic energy consumption. The obtained CRLB also be used for such LED selection to achieve the lowest VLP error within the LED power budget, as per the dependency of CRLB on the active LED set. In addition, our CRLB also builds the theoretical foundation for other VLP system optimization opportunities, e.g., UE orientation optimization, LED selection for minimized VLP error with power constraint, and joint optimization of VLC data transmission and VLP performance. In all these issues, a stochastic optimization-based formulation similar to (51) can be used to handle unknown UE location parameters.

It should be noted that in this section we only aim to provide guidelines for how to improve the VLP system based on the obtained CRLBs, and the design of specific optimization algorithms is out of the scope of this paper.

\section{NUMERICAL RESULTS}

In this section, we present numerical simulations to demonstrate the obtained closed-form result of VLP performance.

\section{A. Simulation Setup}

We consider $M=81$ LED transmitters which are installed, with regular spacing on the ceiling of a room with a size of $9[\mathrm{~m}] \times 9[\mathrm{~m}] \times 4[\mathrm{~m}]$, as shown in Fig. 3 . In addition, we consider 


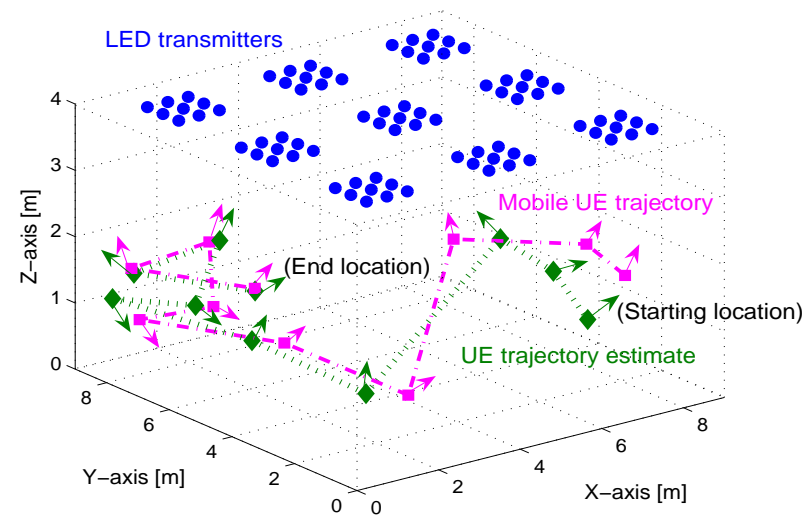

Fig. 3. The LED source layout and the UE trace, where the arrows indicates UE orientations, and a Bayesian filtering-based UE trajectory estimate [52] is shown and used as an example case of vehicular VLP method.

the common case that all LED orientations are downward, i.e., $\mathbf{v}_{m}=[0,0,-1]^{\top}, \forall m=1: M$, although the the obtained CRLB has no special requirement on LED orientation angles. The UE appears in the room at a random location and with a random orientation. In addition, we assume $\theta_{\mathrm{FOV}}=\phi_{\mathrm{FOV}}=$ $\pi / 2$ and $r=1$. These parameter settings follow from a typical LED setup that is widely adopted in papers such as [11], [13], [56], [57]. We consider $\mathrm{SNR}=20[\mathrm{~dB}]$ and $\Psi_{R}=5$ by merging values of transmitting power, optical filter gain and optical concentrator gain, unless specified otherwise. We consider a generic case of UE movement in which $\chi_{\text {trans }}=10^{2} \mathbf{I}_{3}$ and $\mathbf{U}_{\text {trans }}=10^{2} \mathbf{I}_{3}$, and also $\chi_{\text {prior }}=4 \mathbf{I}$ as well as $\mathbf{U}_{\text {prior }}=2 \mathbf{I}_{3}$, unless specified otherwise.

In the following, we shall provide the associated numerical results to demonstrate the obtained performance limits of VLP and the effect of each dominant factor, respectively.

\section{B. Long-Term VLP Performance}

1) Time-Domain VLP Cooperation Gain: The real-time evolution of the VLP error bounds is presented in Fig. 4, where the achieved time-domain VLP cooperation gain of UE location and orientation are highlighted. We can see that the VLP error will reduce over time and then reach its stable state eventually, which complies with theorem 2-4 in section V-B (the statable state variation has been mitigated by the simulation average). The achieved long-term VLP error perfectly coincides with its theoretical stable state.

2) The Impact of UE Mobility and SNR: The real-time VLP error bound and the associated stable state versus SNR and UE transition precision are presented in Fig. 5. The larger SNR and greater transition precision will give rise to a low stable state of the VLP error. This is reasonable because the VLP system will gain more knowledge from a more precise UE location transition as well as a larger SNR.

3) The Impact of Prior Knowledge: The long-term VLP performance versus prior knowledge is presented in Fig. 6. We can see that the achieved long-term VLP error (i.e., $\operatorname{trace}\left(\mathcal{B}_{\mathbf{x}}^{\star}\right)$ ) is perfectly consistent with the obtained theoretical stable state. In addition, it is not surprising that the larger the SNR is, the lower the VLP error becomes. Moreover, the VLP error will

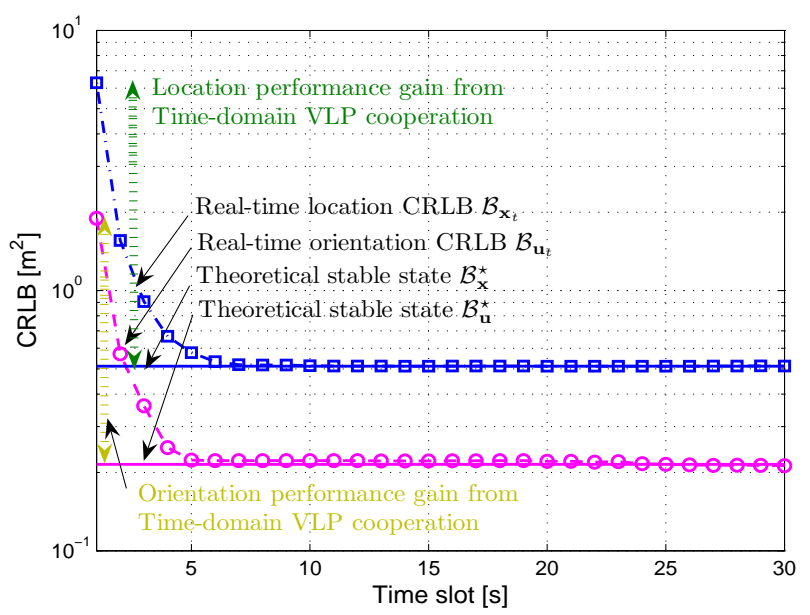

Fig. 4. Real-time CRLB with time-domain VLP cooperation $(\mathrm{SNR}=0 \mathrm{~dB})$.

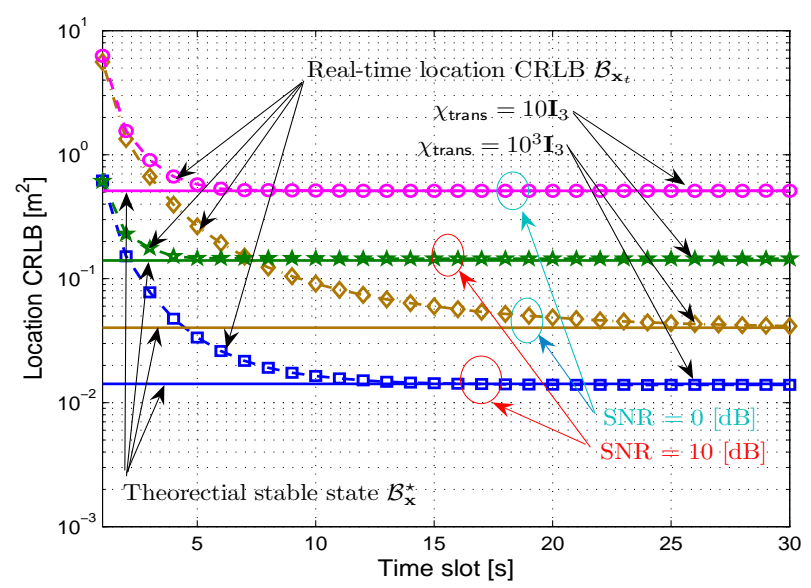

Fig. 5. Real-time CRLB over different settings of SNR and UE mobility.

show an error ceiling phenomenon at the low SNR region, since the VLP performance will be dominated by the prior knowledge of UE location parameters. Naturally, the ceiling of the VLP error depends on the amount of prior knowledge. Specifically, the less prior knowledge gives rise to the larger value of the VLP error ceiling.

\section{CONCLUSIONS}

In this paper, the performance limits of vehicular VLP for mobile UEs are comprehensively studied. Specifically, a closed-form CRLB for the UE location and orientation, respectively, is obtained, which can give insights into the informative contribution of prior knowledge, UE mobility and observation information to the overall VLP performance. Moreover, the time-domain evolution of the VLP error is studied, which sheds light on the long-term VLP performance and helps understand the essence of time-domain localization cooperation. The obtained closed-form CRLBs on UE location and orientation errors can not only serve as a performance benchmark for practical vehicular VLP systems, but also give insights into how system parameters and LED deployment affect the UE location and orientation estimate performance. 


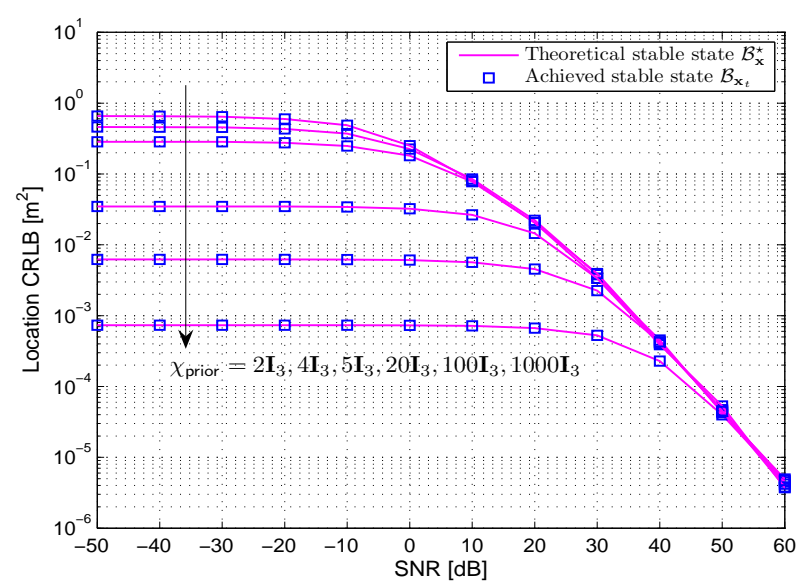

Fig. 6. Long-term VLP error v.s. SNR and prior location knowledge.

Finally, based on the dependency of the obtained closedform CRLBs on the system parameters, the guidelines for VLP system optimization design are provided, which can be used to fundamentally improve the vehicular VLP system performance via resource allocation and system deployment optimization.

\section{APPENDIX A \\ PROOF OF THEOREM 1}

As per the estimate theory [38], the unbiased estimate error of $\boldsymbol{\alpha}_{t}$ will be bounded from below as $\mathbb{E}\left\{\left\|\hat{\boldsymbol{\alpha}}_{t}-\boldsymbol{\alpha}_{t}\right\|_{2}^{2}\right\} \geq$ $\operatorname{trace}\left(\mathcal{B}_{\boldsymbol{\alpha}_{t}}\left(\boldsymbol{\alpha}_{t}\right)\right)$, where $\mathcal{B}_{\boldsymbol{\alpha}_{t}}\left(\boldsymbol{\alpha}_{t}\right) \in \mathbb{S}^{6}$ is its CRLB, given by

$$
\mathcal{B}_{\boldsymbol{\alpha}_{t}}\left(\boldsymbol{\alpha}_{t}\right)=\left(\mathcal{I}_{\boldsymbol{\alpha}_{t}}\left(\boldsymbol{\alpha}_{t}\right)\right)^{-1}
$$

where $\mathcal{I}_{\boldsymbol{\alpha}_{t}}\left(\boldsymbol{\alpha}_{t}\right)$ is the FIM that is defined as [38]

$$
\mathcal{I}_{\boldsymbol{\alpha}_{t}}\left(\boldsymbol{\alpha}_{t}\right)=-\mathbb{E}_{\boldsymbol{\alpha}_{t} \mid \mathbf{z}_{1: t}}\left\{\nabla_{\boldsymbol{\alpha}_{t}, \boldsymbol{\alpha}_{t}^{\top}} \ln p\left(\boldsymbol{\alpha}_{t} \mid \mathbf{z}_{1: t}, \widetilde{\boldsymbol{\alpha}}_{1: t}\right)\right\},
$$

where $\nabla_{\boldsymbol{\alpha}_{t}, \boldsymbol{\alpha}_{t}^{\top}}(\bullet)$ is the second-order derivative w.r.t. $\boldsymbol{\alpha}_{t}$, and $p\left(\boldsymbol{\alpha}_{t} \mid \mathbf{z}_{1: t}\right)$ is the posterior given in (13).

Based on (13), we know the posterior $p\left(\boldsymbol{\alpha}_{t} \mid \mathbf{z}_{1: t}, \widetilde{\boldsymbol{\alpha}}_{1: t}\right) \propto$ $p\left(\mathbf{z}_{t} \mid \boldsymbol{\alpha}_{t}\right) p\left(\boldsymbol{\alpha}_{t} \mid \mathbf{z}_{1: t-1}, \widetilde{\boldsymbol{\alpha}}_{1: t-1}\right) p\left(\boldsymbol{\alpha}_{t} \mid \widetilde{\boldsymbol{\alpha}}_{t}\right)$, and the FIM can be partitioned into two parts, namely,

$$
\mathcal{I}_{\boldsymbol{\alpha}_{t}}\left(\boldsymbol{\alpha}_{t}\right)=\mathcal{I}_{\boldsymbol{\alpha}_{t}}^{\text {obs }}\left(\boldsymbol{\alpha}_{t}\right)+\mathcal{I}_{\boldsymbol{\alpha}_{t}}^{\text {pred }}\left(\boldsymbol{\alpha}_{t}\right)
$$

where the observation information matrix $\mathcal{I}_{\boldsymbol{\alpha}_{t}}^{\text {obs }}\left(\boldsymbol{\alpha}_{t}\right)$ related to $p\left(\mathbf{z}_{t} \mid \boldsymbol{\alpha}_{t}\right)$ and the prediction information matrix $\mathcal{I}_{\boldsymbol{\alpha}_{t}}^{\text {pred }}\left(\boldsymbol{\alpha}_{t}\right)$ regarding $p\left(\boldsymbol{\alpha}_{t} \mid \mathbf{z}_{1: t-1}, \widetilde{\boldsymbol{\alpha}}_{1: t-1}\right) p\left(\boldsymbol{\alpha}_{t} \mid \widetilde{\boldsymbol{\alpha}}_{t}\right)$ are given by

$$
\begin{aligned}
\mathcal{I}_{\boldsymbol{\alpha}_{t}}^{\text {obs }}\left(\boldsymbol{\alpha}_{t}\right) & =-\mathbb{E}\left\{\nabla_{\boldsymbol{\alpha}_{t}, \boldsymbol{\alpha}_{t}^{\top}} \ln p\left(\mathbf{z}_{t} \mid \boldsymbol{\alpha}_{t}\right)\right\}, \\
\mathcal{I}_{\boldsymbol{\alpha}_{t}}^{\text {pred }}\left(\boldsymbol{\alpha}_{t}\right) & =-\mathbb{E}\left\{\nabla_{\boldsymbol{\alpha}_{t}, \boldsymbol{\alpha}_{t}^{\top}} \ln \left(p\left(\boldsymbol{\alpha}_{t} \mid \mathbf{z}_{1: t-1}, \widetilde{\boldsymbol{\alpha}}_{1: t-1}\right) p\left(\boldsymbol{\alpha}_{t} \mid \widetilde{\boldsymbol{\alpha}}_{t}\right)\right)\right\} .
\end{aligned}
$$

Furthermore, based on the structure of $\boldsymbol{\alpha}_{t}=\left[\mathbf{x}_{t} ; \mathbf{u}_{t}\right]$, the observation FIM can be structured as

$$
\mathcal{I}_{\boldsymbol{\alpha}_{t}}^{\mathrm{obs}}\left(\boldsymbol{\alpha}_{t}\right)=\sum_{m \in \Omega_{t}}\left[\begin{array}{ll}
\mathcal{I}_{\mathbf{x}_{t}, \mathbf{x}_{t} ; m}^{\mathrm{obs}}\left(\boldsymbol{\alpha}_{t}\right) & \mathcal{I}_{\mathbf{x}_{t}, \mathbf{u}_{t} ; m}^{\text {obs }}\left(\boldsymbol{\alpha}_{t}\right) \\
\mathcal{I}_{\mathbf{u}_{t}, \mathbf{x}_{t} ; m}^{\mathrm{obs}}\left(\boldsymbol{\alpha}_{t}\right) & \mathcal{I}_{\mathbf{u}_{t}, \mathbf{u}_{t} ; m}^{\mathrm{obs}}\left(\boldsymbol{\alpha}_{t}\right)
\end{array}\right],
$$

where $\mathcal{I}_{\boldsymbol{\alpha}_{i, t}, \boldsymbol{\alpha}_{j, t} ; m}^{\text {obs }}\left(\boldsymbol{\alpha}_{t}\right) \in \mathbb{R}^{3 \times 3}$, for $\boldsymbol{\alpha}_{i, t}, \boldsymbol{\alpha}_{j, t} \in\left\{\mathbf{x}_{t}, \mathbf{u}_{t}\right\}$, is the elementary cross FIM of observation given by

$$
\mathcal{I}_{m ; \boldsymbol{\alpha}_{i, t}, \boldsymbol{\alpha}_{j, t}}^{\mathrm{obs}}\left(\boldsymbol{\alpha}_{t}\right)=-\mathbb{E}\left\{\nabla_{\boldsymbol{\alpha}_{i, t}, \boldsymbol{\alpha}_{j, t}^{\top}} \ln p\left(\mathrm{z}_{m, t} \mid \boldsymbol{\alpha}_{t}\right)\right\} .
$$

In the following, we shall explicate the prediction FIM $\mathcal{I}_{\boldsymbol{\alpha}_{t}}^{\text {pred }}\left(\boldsymbol{\alpha}_{t}\right)$ and observation FIM $\mathcal{I}_{\boldsymbol{\alpha}_{t}}^{\text {obs }}\left(\boldsymbol{\alpha}_{t}\right)$, respectively.

1) Prediction FIM: Let $\hat{\mathbf{x}}_{t-1}$ and $\hat{\mathbf{u}}_{t-1}$ be the previous posterior estimates of UE location and orientation, respectively, at time slot $t-1$, and let $\chi_{t-1}^{\sharp}$ and $\mathbf{U}_{t-1}^{\sharp}$ be their posterior precision matrices, respectively, which can be fully determined by the previous posterior function $p\left(\mathbf{x}_{t-1}, \mathbf{u}_{t-1} \mid \mathbf{z}_{1: t-1}\right)$.

We use a Gaussian kernel to extract principal information of the previous posterior: $p\left(\mathbf{x}_{t-1}, \mathbf{u}_{t-1} \mid \mathbf{z}_{1: t-1}, \widetilde{\boldsymbol{\alpha}}_{1: t-1}\right) \approx$ $\mathcal{N}\left(\mathbf{x}_{t-1} \mid \hat{\mathbf{x}}_{t-1}, \chi_{t-1}^{\sharp}\right) \mathcal{N}\left(\mathbf{u}_{t-1} \mid \hat{\mathbf{u}}_{t-1}, \mathbf{U}_{t-1}^{\sharp}\right)$, where ${ }^{7}$

$$
\begin{aligned}
\hat{\boldsymbol{\alpha}}_{t-1} & =\underset{\boldsymbol{\alpha}_{t-1}}{\arg \max } \ln p\left(\boldsymbol{\alpha}_{t-1} \mid \mathbf{z}_{1: t-1}, \widetilde{\boldsymbol{\alpha}}_{1: t-1}\right), \\
\mathbf{W}_{t-1}^{\sharp} & =\left.\nabla_{\boldsymbol{\alpha}_{t-1}, \boldsymbol{\alpha}_{t-1}^{\top}} \ln p\left(\boldsymbol{\alpha}_{t-1} \mid \mathbf{z}_{1: t-1}, \widetilde{\boldsymbol{\alpha}}_{1: t-1}\right)\right|_{\boldsymbol{\alpha}_{t-1}=\hat{\boldsymbol{\alpha}}_{t-1}}
\end{aligned}
$$

while $\chi_{t-1}^{\sharp}=\left[\mathbf{W}_{t-1}^{\sharp}\right]_{1: 3,1: 3}$ and $\mathbf{U}_{t-1}^{\sharp}=\left[\mathbf{W}_{t-1}^{\sharp}\right]_{4: 6,4: 6}$.

Thus, the prediction distribution will eventually follow that

$$
\begin{aligned}
& p\left(\mathbf{x}_{t}, \mathbf{u}_{t} \mid \mathbf{z}_{1: t-1}, \widetilde{\boldsymbol{\alpha}}_{1: t-1}\right) \\
= & \mathcal{N}\left(\mathbf{x}_{t} \mid \mathbf{x}_{1: t-1}, \boldsymbol{\chi}_{\text {pred }, t}^{\prime}\right) \mathcal{N}\left(\mathbf{u}_{t} \mid \mathbf{u}_{1: t-1}, \mathbf{U}_{\text {pred }, t}^{\prime}\right),
\end{aligned}
$$

where $\mathbf{x}_{1: t-1}$ and $\mathbf{u}_{1: t-1}$ are the prediction means, while $\chi_{\text {pred }}^{\prime}$ and $\mathbf{U}_{\text {pred }}^{\prime}$ are the associated prediction precisions, given by

$$
\begin{array}{r}
\mathbf{x}_{1: t-1}=\left(\boldsymbol{\chi}_{\text {pred }, t}^{\prime}\right)^{-1}\left(\chi_{\text {pred }, t} \hat{\mathbf{x}}_{t-1}+\chi_{\text {prior }} \widetilde{\mathbf{x}}_{t}\right), \\
\mathbf{u}_{1: t-1}=\left(\mathbf{U}_{\text {pred }, t}^{\prime}\right)^{-1}\left(\mathbf{U}_{\text {pred }, t} \hat{\mathbf{u}}_{t-1}+\mathbf{U}_{\text {prior }} \widetilde{\mathbf{u}}_{t}\right) \\
\chi_{\text {pred }, t}^{\prime}=\chi_{\text {prior }}+\underbrace{\left(\chi_{\text {trans }}^{-1}+\left(\boldsymbol{\chi}_{t-1}^{\sharp}\right)^{-1}\right)^{-1}}_{\boldsymbol{\chi}_{\text {pred }, t}}, \\
\mathbf{U}_{\text {pred }, t}^{\prime}=\mathbf{U}_{\text {prior }}+\underbrace{\left(\mathbf{U}_{\text {trans }}^{-1}+\left(\mathbf{U}_{t-1}^{\#}\right)^{-1}\right)^{-1}}_{\mathbf{U}_{\text {pred }, t}} .
\end{array}
$$

Note that $\left(\chi_{t-1}^{\sharp}\right)^{-1} \succeq \mathcal{B}_{\mathbf{x}_{t-1}}\left(\boldsymbol{\alpha}_{t-1}\right)$ as per estimate theory, and thus $\chi_{\text {pred }, t}^{\prime} \preceq \underbrace{\chi_{\text {prior }}+\left(\chi_{\text {trans }}^{-1}+\mathcal{B}_{\mathbf{x}_{t-1}}\left(\boldsymbol{\alpha}_{t-1}\right)\right)^{-1}}_{\chi_{\text {pred }, t}^{\circ}}$, and so does $\mathbf{U}_{\text {pred, } t}^{\prime}$ lower than $\mathbf{U}_{\text {pred, }, t}^{\circ}$. That means, the relaxation from $\left(\chi_{t-1}^{\sharp}\right)^{-1}$ to $\mathcal{B}_{\mathbf{x}_{t-1}}\left(\boldsymbol{\alpha}_{t-1}\right)$ leads to a lower approximation for $\mathcal{B}_{\mathbf{x}_{t}}\left(\boldsymbol{\alpha}_{t}\right)$. Thus, this approximation will not change the direction of the CRLB inequality. Hence, as per (16), we can easily arrive at $\mathcal{I}_{\boldsymbol{\alpha}_{t}}^{\text {pred }}\left(\boldsymbol{\alpha}_{t}\right)=\left[\begin{array}{cc}\chi_{\text {pred }, t}^{\circ} & \mathbf{0} \\ \mathbf{0} & \mathbf{U}_{\text {pred }, t}^{\circ}\end{array}\right]$.

2) Observation FIM: We shall explicate each observation FIM $\mathcal{I}_{\boldsymbol{\alpha}_{i, t}, \boldsymbol{\alpha}_{j, t} ; m}^{\text {obs }}\left(\boldsymbol{\alpha}_{t}\right), \forall \boldsymbol{\alpha}_{i, t}, \boldsymbol{\alpha}_{j, t} \in\left\{\mathbf{x}_{t}, \mathbf{u}_{t}\right\}$.

Firstly, we consider $\mathcal{I}_{\mathbf{x}_{t}, \mathbf{x}_{t} ; m}^{\text {obs }}\left(\boldsymbol{\alpha}_{t}\right)$. Based on the system model, we have $\nabla_{\mathbf{x}_{t}} h_{m}\left(\mathbf{x}_{t}, \mathbf{u}_{t}\right)$ formed as

$$
\nabla_{\mathbf{x}_{t}} h_{m}\left(\mathbf{x}_{t}, \mathbf{u}_{t}\right)=\Psi_{R} \rho_{m, t}^{-3} \mathbf{D}_{m}\left(\mathbf{x}_{t}\right) \frac{\mathbf{u}_{t}}{\left\|\mathbf{u}_{t}\right\|_{2}}
$$

where $\mathbf{D}_{m}\left(\mathbf{x}_{t}\right) \in \mathbb{R}^{3 \times 3}$ is given by (27). Then, we arrive at $\mathcal{I}_{m ; \mathbf{x}_{t}, \mathbf{x}_{t}}^{\text {obs }}\left(\boldsymbol{\alpha}_{t}\right)=\operatorname{SNR} \rho_{m, t}^{-6} \mathbf{D}_{m}\left(\mathbf{x}_{t}\right) \frac{\mathbf{u}_{t} \mathbf{u}_{t}^{\top}}{\left\|\mathbf{u}_{t}\right\|_{2}^{2}} \mathbf{D}_{m}^{\top}\left(\mathbf{x}_{t}\right)$.

Secondly, for $\mathcal{I}_{\mathbf{x}_{t}, \mathbf{u}_{t} ; m}^{\text {obs }}\left(\boldsymbol{\alpha}_{t}\right), h_{m}\left(\mathbf{x}_{t}, \mathbf{u}_{t}\right)$ can be rewritten as

${ }^{7}$ This approximation can preserve principal information of the original distribution (leading to the minimum entropy difference, given the same mean and precision) as per the Laplacian approximation [45], [46]. 
a compact function of $\mathbf{u}_{t}$ as follows,

$$
h_{m}\left(\mathbf{x}_{t}, \mathbf{u}_{t}\right)=\Psi_{R} \rho_{m, t}^{-2} \mathbf{d}_{m, t}^{\top} \frac{\mathbf{u}_{t}}{\left\|\mathbf{u}_{t}\right\|_{2}},
$$

where $\mathbf{d}_{m, t}=(r+1)\left(\mathbf{e}_{m, t}^{\top} \mathbf{v}_{m}\right)^{r} \mathbf{e}_{m, t}$. Thus, $\nabla_{\mathbf{u}_{t}} h_{m}\left(\mathbf{x}_{t}, \mathbf{u}_{t}\right)$ can be derived as

$$
\nabla_{\mathbf{u}_{t}} h_{m}\left(\mathbf{x}_{t}, \mathbf{u}_{t}\right)=\Psi_{R} \rho_{m, t}^{-2}(\underbrace{\frac{\left\|\mathbf{u}_{t}\right\|_{2}^{2} \mathbf{I}_{3}-\mathbf{u}_{t} \mathbf{u}_{t}^{\top}}{\left\|\mathbf{u}_{t}\right\|_{2}^{3}}}_{\mathcal{R}_{t}}) \mathbf{d}_{m, t} .
$$

Hence, $\mathcal{I}_{\mathbf{x}_{t}, \mathbf{u}_{t} ; m}^{\text {obs }}\left(\boldsymbol{\alpha}_{t}\right)=\operatorname{SNR} \rho_{m, t}^{-5} \mathbf{D}_{m}\left(\mathbf{x}_{t}\right) \frac{\mathbf{u}_{t}}{\left\|\mathbf{u}_{t}\right\|_{2}} \mathbf{d}_{m, t}^{\top} \boldsymbol{\mathcal { R }}_{t}^{\top}$ as per (59). Note that $\mathcal{I}_{\mathbf{u}_{t}, \mathbf{x}_{t} ; m}\left(\boldsymbol{\alpha}_{t}\right)=\left(\mathcal{I}_{\mathbf{x}_{t}, \mathbf{u}_{t} ; m}^{\text {obs }}\left(\boldsymbol{\alpha}_{t}\right)\right)^{\top}$.

Thirdly, for $\mathcal{I}_{\mathbf{u}_{t}, \mathbf{u}_{t} ; m}^{\text {obs }}\left(\boldsymbol{\alpha}_{t}\right)$, based on (68) and (59) we can obtain $\mathcal{I}_{m ; \mathbf{u}_{t}, \mathbf{u}_{t}}^{\text {obs }}\left(\boldsymbol{\alpha}_{t}\right)=\operatorname{SNR} \rho_{m, t}^{-4} \boldsymbol{\mathcal { R }}_{t} \mathbf{d}_{m, t} \mathbf{d}_{m, t}^{\top} \boldsymbol{\mathcal { R }}_{t}^{\top}$.

Hence, $\mathcal{I}_{\boldsymbol{\alpha}_{t}}^{\text {obs }}\left(\boldsymbol{\alpha}_{t}\right)$ is obtained. Then, based on the FIM structure in (57) and (58) and combing it with $\mathbf{K}\left(\mathbf{u}_{t}\right)$ and $\mathbf{H}\left(\mathbf{x}_{t}\right)$ in (26), the joint CRLB $\mathcal{B}_{\boldsymbol{\alpha}_{t}}\left(\boldsymbol{\alpha}_{t}\right)$ is give by (69). Then, applying the Schur complement [53], the equivalent FIM $\mathcal{J}_{\mathbf{x}_{t}}\left(\mathbf{x}_{t}, \mathbf{u}_{t}\right)$ and $\mathcal{J}_{\mathbf{u}_{t}}\left(\mathbf{x}_{t}, \mathbf{u}_{t}\right)$ can be cast as (24) and (25), respectively. Thus, theorem 2 is proved.

\section{APPENDIX B \\ PROOF OF THEOREM 2}

The proof is comprised of two parts. The first regards the convergence behavior of TDEE, and the second regards the associated convergence rate.

1) Proof Of Convergence: For the convergence of TDEE, the crux is to prove the iteration of TDEE is contractive.

For any two semi-definite positive CRLB matrices $\mathcal{B}_{\mathbf{x}_{t-1}}^{(\mathrm{a})}$ and $\mathcal{B}_{\mathbf{x}_{t-1}}^{(\mathrm{b})}$ in $\mathbb{S}_{+}^{3}$ with $\mathcal{B}_{\mathbf{x}_{t-1}}^{(\mathrm{a})} \succeq \mathcal{B}_{\mathbf{x}_{t-1}}^{(\mathrm{b})}$, we need to prove the following contraction inequality always holds,

$$
\left\|\mathcal{B}_{\mathbf{x}_{t}}^{(\mathrm{a})}-\mathcal{B}_{\mathbf{x}_{t}}^{(\mathrm{b})}\right\|_{F} \leq\left\|\mathcal{B}_{\mathbf{x}_{t-1}}^{(\mathrm{a})}-\mathcal{B}_{\mathbf{x}_{t-1}}^{(\mathrm{b})}\right\|_{F} .
$$

Combining (70) with (32), we need to prove

$$
\begin{aligned}
& \left\|\left(\mathbf{C}_{t}+\left(\chi_{\text {trans }}^{-1}+\mathcal{B}_{\mathbf{x}_{t-1}}^{(\mathrm{a})}\right)^{-1}\right)^{-1}-\left(\mathbf{C}_{t}+\left(\chi_{\text {trans }}^{-1}+\mathcal{B}_{\mathbf{x}_{t-1}}^{(\mathrm{b})}\right)^{-1}\right)^{-1}\right\|_{F} \\
\leq & \left\|\mathcal{B}_{\mathbf{x}_{t-1}}^{(\mathrm{a})}-\mathcal{B}_{\mathbf{x}_{t-1}}^{(\mathrm{b})}\right\|_{F},
\end{aligned}
$$

where we use $\mathbf{C}_{t}$ to denote $\mathcal{H}_{\mathbf{x}_{t}}^{\text {obs }}+\chi_{\text {prior }}$ for brevity. Applying the inverse matrix lemma, we have (72). Thus, the inequality in (71) we need to prove can be rewritten as

$$
\left\|\mathcal{B}_{\mathbf{x}_{t-1}}^{(\mathrm{a})}-\mathcal{B}_{\mathbf{x}_{t-1}}^{(\mathrm{b})}-\mathbf{Q}_{t}\right\|_{F} \leq\left\|\mathcal{B}_{\mathbf{x}_{t-1}}^{(\mathrm{a})}-\mathcal{B}_{\mathbf{x}_{t-1}}^{(\mathrm{b})}\right\|_{F},
$$

where $\mathbf{Q}_{t}=\left(\left(\chi_{\text {trans }}^{-1}+\mathcal{B}_{\mathbf{x}_{t-1}}^{(\mathrm{a})}\right)^{-1} \mathbf{C}_{t}^{-1}\left(\chi_{\text {trans }}^{-1}+\mathcal{B}_{\mathbf{x}_{t-1}}^{(\mathrm{a})}\right)^{-1}+\right.$ $\left.\left(\chi_{\text {trans }}^{-1}+\mathcal{B}_{\mathbf{x}_{t-1}}^{(\mathrm{a})}\right)^{-1}\right)^{-1}-\left(\left(\chi_{\text {trans }}^{-1}+\mathcal{B}_{\mathbf{x}_{t-1}}^{(\mathrm{b})}\right)^{-1} \mathbf{C}_{t}^{-1}\left(\chi_{\text {trans }}^{-1}+\right.\right.$ $\left.\left.\mathcal{B}_{\mathbf{x}_{t-1}}^{(\mathrm{b})}\right)^{-1}+\left(\chi_{\text {trans }}^{-1}+\mathcal{B}_{\mathbf{x}_{t-1}}^{(\mathrm{b})}\right)^{-1}\right)^{-1}$.

Since we have assumed $\mathcal{B}_{\mathbf{x}_{t-1}}^{(\mathrm{a})} \succeq \mathcal{B}_{\mathbf{x}_{t-1}}^{(\mathrm{b})}$ and $\mathbf{C}_{t}$ as well as $\boldsymbol{\chi}_{\text {trans }}^{-1}$ is semi-definite positive, $\mathcal{B}_{\mathbf{x}_{t-1}}^{(\mathrm{a})}-\mathcal{B}_{\mathbf{x}_{t-1}}^{(\mathrm{b})} \succeq \mathbf{0}$ and $\mathbf{Q}_{t} \succeq \mathbf{0}$ must hold. Hence, (73) is satisfied. As a result, the contraction inequality in (70) always holds, and this proof can be easily extended to the contraction inequality for $\mathcal{B}_{\mathbf{u}_{t}}$.

2) Convergence Rate: Applying the inverse matrix lemma for (32), we have $\mathcal{B}_{\mathbf{x}_{t}}-\mathcal{B}_{\mathbf{x}_{t+1}}=\mathcal{B}_{\mathbf{x}_{t-1}}-\mathcal{B}_{\mathbf{x}_{t}}-\mathbf{Q}_{t}^{\prime}$, where $\mathbf{Q}_{t}^{\prime}$ is given by (74), and we know $\mathbf{Q}_{t}^{\prime} \succeq \mathbf{0}$. Let $\eta_{\mathbf{x}}$ be the convergence rate of $\boldsymbol{B}_{\mathbf{x}_{t}}$. Then, we have $\eta_{\mathbf{x}}=$ $\lim _{t \rightarrow \infty} \frac{\left\|\mathcal{B}_{\mathbf{x}_{t}}-\mathcal{B}_{\mathbf{x}_{t+1}}\right\|_{F}}{\left\|\mathcal{B}_{\mathbf{x}_{t-1}}-\mathcal{B}_{\mathbf{x}_{t}}\right\|_{F}}=\lim _{t \rightarrow \infty} \frac{\left\|\mathcal{B}_{\mathbf{x}_{t-1}}-\mathcal{B}_{\mathbf{x}_{t}}-\mathbf{Q}_{t}\right\|_{F}}{\left\|\mathcal{B}_{\mathbf{x}_{t-1}}-\mathcal{B}_{\mathbf{x}_{t}}\right\|_{F}}$. Hence, we have $\eta_{\mathbf{x}} \in\left(1-\kappa_{\mathbf{x}}, 1\right)$ with $\kappa_{\mathbf{x}}=\lim _{t \rightarrow \infty} \frac{\left\|\mathbf{Q}_{t}^{\prime}\right\|_{F}}{\left\|\mathcal{B}_{\mathbf{x}_{t}}-\mathcal{B}_{\mathbf{x}_{t-1}}\right\|_{F}}$, since $\mathbf{Q}_{t}^{\prime} \succeq \mathbf{0}$. After some algebra, $\kappa_{\mathbf{x}}$ can be eventually cast as (38), where $\mathcal{H}_{\mathbf{x} \star}^{\sharp}$ has been given by (34), and hence we have $\kappa_{\mathbf{x}} \in(0,1)$. Then, $\eta_{\mathbf{x}}=1-\kappa_{\mathbf{x}}$ is within $(0,1)$, and so is $\eta_{\mathbf{u}}$. Thus, theorem 5 is proved.

\section{APPENDIX C \\ PROOF OF THEOREM 3}

Since TDEE is identical to a fixed-point iteration, $\mathcal{B}_{\mathrm{x}}^{\star}$ is the fixed-point solution of the error evolution function, i.e.,

$$
\begin{aligned}
& \mathcal{B}_{\mathbf{x}}^{\star}=\left(\left(\mathcal{H}_{\mathbf{x} \star}\right)^{\sharp}+\left(\boldsymbol{\chi}_{\text {trans }}^{-1}+\mathcal{B}_{\mathbf{x}}^{\star}\right)^{-1}\right)^{-1}, \\
& \mathcal{B}_{\mathbf{u}}^{\star}=\left(\left(\mathcal{H}_{\mathbf{u} \star}\right)^{\sharp}+\left(\mathbf{U}_{\text {trans }}^{-1}+\mathcal{B}_{\mathbf{u}}^{\star}\right)^{-1}\right)^{-1} .
\end{aligned}
$$

Applying the inverse matrix lemma, the above fixed-point equations can be equivalently rewritten as

$$
\begin{aligned}
& \left(\mathcal{B}_{\mathbf{x}}^{\star}\right)^{\top} \boldsymbol{\chi}_{\text {trans }} \mathcal{B}_{\mathbf{x}}^{\star}+\mathcal{B}_{\mathbf{x}}^{\star}=\left(\mathcal{H}_{\mathbf{x} \star}^{\sharp}\right)^{-1}, \\
& \left(\mathcal{B}_{\mathbf{u}}^{\star}\right)^{\top} \mathbf{U}_{\text {trans }} \mathcal{B}_{\mathbf{u}}^{\star}+\mathcal{B}_{\mathbf{u}}^{\star}=\left(\mathcal{H}_{\mathbf{u} \star}^{\sharp}\right)^{-1} .
\end{aligned}
$$

Hence, we should verify that the closed-form solution of the stable state satisfies the above fixed-point equation. In addition, $\mathcal{B}_{\mathbf{x}}^{\star}$ follows (79). Thus, we can see that $\mathcal{B}_{\mathbf{x}}^{\star}$ satisfies (77), and hence it is the fixed-point solution of (32). We can also prove that $\mathcal{B}_{\mathbf{u}}^{\star}$ is the fixed-point solution of (33). Theorem 3 is proved.

\section{APPENDIX D \\ PROOF OF THEOREM 4}

Firstly, since we assume no measurement at initial time $t=$ 0 , we have $\mathcal{J}_{\mathbf{x}_{0}}=\mathbf{0}$. Thus, for $t=1$, we have $\boldsymbol{\chi}_{\text {pred, } 1}=\mathbf{0}$ and hence $\mathcal{B}_{\mathbf{x}_{1}}=\left(\mathcal{H}_{\mathbf{x}_{1}}^{\text {obs }}+\boldsymbol{\chi}_{\text {prior }}\right)^{-1}$. In addition, for $t=2$ we have $\mathcal{B}_{\mathbf{x}_{2}}=\left(\mathcal{H}_{\mathbf{x}_{2}}^{\text {obs }}+\boldsymbol{\chi}_{\text {prior }}+\boldsymbol{\chi}_{\text {pred }, 2}\right)^{-1}$. Given condition B1 that $\mathcal{H}_{\mathbf{x}_{2}}^{\text {obs }} \succeq \mathcal{H}_{\mathbf{x}_{1}}^{\text {obs }}$, we obtain $\mathcal{B}_{\mathbf{x}_{2}} \preceq \mathcal{B}_{\mathbf{x}_{1}}$ since $\boldsymbol{\chi}_{\text {pred }, 2} \succeq \mathbf{0}$.

To completely prove theorem 7 , in the following we shall prove $\mathcal{B}_{\mathbf{x}_{t+1}} \preceq \mathcal{B}_{\mathbf{x}_{t}}$ holds if $\mathcal{B}_{\mathbf{x}_{t}} \preceq \mathcal{B}_{\mathbf{x}_{t-1}}$. We know $\mathcal{B}_{\mathbf{x}_{t}}=$ $\left(\mathcal{H}_{\mathbf{x}_{t}}^{\text {obs }}+\boldsymbol{\chi}_{\text {prior }}+\left(\boldsymbol{B}_{\mathbf{x}_{t}}+\boldsymbol{\chi}_{\text {trans }}^{-1}\right)^{-1}\right)^{-1}$. If $\boldsymbol{\mathcal { B }}_{\mathbf{x}_{t}} \preceq \boldsymbol{B}_{\mathbf{x}_{t-1}}$ holds and $\mathrm{B} 1$ is satisfied, we must have $\mathcal{B}_{\mathbf{x}_{t+1}} \preceq \mathcal{B}_{\mathbf{x}_{t}}$. Thus, theorem 4 is proved.

\section{REFERENCES}

[1] A. Jovicic, J. Li and T. Richardson, "Visible Light Communication: Opportunities, Challenges and the Path to Market," IEEE Communications Magazine, Vol.51, No.12, 2013, pp.26-32.

[2] Y. S. Kuo, P. Pannuto, K. J. Hsiao, and P. Dutta, "Luxapose: Indoor positioning with mobile phones and visible light," Proceedings of the 20th annual international conference on Mobile computing and networking. ACM, 2014

[3] Y. Zhuang, L. Hua, L. Qi, J. Yang, P. Cao, Y. Cao, Y. Wu, J. Thompson, and H. Haas, "A Survey of Positioning Systems Using Visible LED Lights," IEEE Communications Surveys \& Tutorials, Vol.20, No.3, 2018, pp.1963-1988.

[4] S. H. Yang, H. S. Kim, Y. H. Son and S. K. Han, "Three-Dimensional Visible Light Indoor Localization Using AOA and RSS With Multiple Optical Receivers," Journal of Lightwave Technology, Vol.32, No.14, 2014, pp.2480-2485.

[5] T. Komine and M. Nakagawa, "Fundamental analysis for visible-light communication system using LED lights," IEEE Trans. on Consumer Electronics, Vol.50, No.1, pp.100-107, Feb 2004. 


$$
\mathcal{B}_{\boldsymbol{\alpha}_{t}}\left(\boldsymbol{\alpha}_{t}\right)=\left(\left[\begin{array}{ll}
\operatorname{SNR} \cdot \mathbf{H}\left(\mathbf{x}_{t}\right) \mathbf{K}\left(\mathbf{u}_{t}\right)\left(\mathbf{K}\left(\mathbf{u}_{t}\right)\right)^{\top}\left(\mathbf{H}\left(\mathbf{x}_{t}\right)\right)^{\top}+\boldsymbol{\chi}_{\text {pred }, t}^{\circ} & \operatorname{SNR} \cdot \mathbf{H}\left(\mathbf{x}_{t}\right) \mathbf{K}\left(\mathbf{u}_{t}\right) \mathbf{G}\left(\mathbf{x}_{t}\right) \boldsymbol{\mathcal { R }}_{t}^{\top} \\
\operatorname{SNR} \cdot \mathcal{R}_{t}\left(\mathbf{G}\left(\mathbf{x}_{t}\right)\right)^{\top}\left(\mathbf{K}\left(\mathbf{u}_{t}\right)\right)^{\top}\left(\mathbf{H}\left(\mathbf{x}_{t}\right)\right)^{\top} & \operatorname{SNR} \cdot \boldsymbol{\mathcal { R }}_{t}\left(\mathbf{G}\left(\mathbf{x}_{t}\right)\right)^{\top} \mathbf{G}\left(\mathbf{x}_{t}\right) \boldsymbol{\mathcal { R }}_{t}^{\top}+\mathbf{U}_{\text {pred }, t}^{\circ}
\end{array}\right]\right)^{-1}
$$

$$
\left(\mathbf{C}_{t}+\left(\boldsymbol{\chi}_{\text {trans }}^{-1}+\mathcal{B}_{\mathbf{x}_{t-1}}^{(\mathrm{a})}\right)^{-1}\right)^{-1}=\boldsymbol{\chi}_{\text {trans }}^{-1}+\mathcal{B}_{\mathbf{x}_{t-1}}^{(\mathrm{a})}-\left(\left(\boldsymbol{\chi}_{\text {trans }}^{-1}+\mathcal{B}_{\mathbf{x}_{t-1}}^{(\mathrm{a})}\right)^{-1} \mathbf{C}_{t}^{-1}\left(\chi_{\text {trans }}^{-1}+\mathcal{B}_{\mathbf{x}_{t-1}}^{(\mathrm{a})}\right)^{-1}+\left(\chi_{\text {trans }}^{-1}+\mathcal{B}_{\mathbf{x}_{t-1}}^{(\mathrm{a})}\right)^{-1}\right)^{-1}
$$

$$
\begin{aligned}
\mathbf{Q}_{t}^{\prime}= & \left(\left(\boldsymbol{\chi}_{\text {trans }}^{-1}+\mathcal{B}_{\mathbf{x}_{t-1}}\right)^{-1} \mathbf{C}_{t}^{-1}\left(\chi_{\text {trans }}^{-1}+\mathcal{B}_{\mathbf{x}_{t-1}}\right)^{-1}+\left(\chi_{\text {trans }}^{-1}+\mathcal{B}_{\mathbf{x}_{t-1}}\right)^{-1}\right)^{-1} \\
& -\left(\left(\chi_{\text {trans }}^{-1}+\mathcal{B}_{\mathbf{x}_{t}}\right)^{-1} \mathbf{C}_{t+1}^{-1}\left(\chi_{\text {trans }}^{-1}+\mathcal{B}_{\mathbf{x}_{t}}\right)^{-1}+\left(\chi_{\text {trans }}^{-1}+\mathcal{B}_{\mathbf{x}_{t}}\right)^{-1}\right)^{-1}
\end{aligned}
$$

$$
\begin{aligned}
\left(\mathcal{B}_{\mathbf{x}}^{\star}\right)^{\top} \boldsymbol{\chi}_{\text {trans }} \mathcal{B}_{\mathbf{x}}^{\star} & =\frac{1}{4} \chi_{\text {trans }}^{-1}-\frac{1}{2} \chi_{\text {trans }}^{-\frac{1}{2}}\left(\mathbf{I}_{3}+4 \boldsymbol{\chi}_{\text {trans }}^{\frac{1}{2}}\left(\mathcal{H}_{\mathbf{x} \star}^{\sharp}\right)^{-1} \chi_{\text {trans }}^{\frac{1}{2}}\right)^{\frac{1}{2}} \chi_{\text {trans }}^{-\frac{1}{2}}+\frac{1}{4} \chi_{\text {trans }}^{-\frac{1}{2}}\left(\mathbf{I}_{3}+4 \chi_{\text {trans }}^{\frac{1}{2}}\left(\mathcal{H}_{\mathbf{x} \star}^{\sharp}\right)^{-1} \chi_{\text {trans }}^{\frac{1}{2}}\right) \chi_{\text {trans }}^{-\frac{1}{2}} \\
& =\frac{1}{2} \chi_{\text {trans }}^{-1}-\frac{1}{2} \chi_{\text {trans }}^{-\frac{1}{2}}\left(\mathbf{I}_{3}+4 \boldsymbol{\chi}_{\text {trans }}^{\frac{1}{2}}\left(\mathcal{H}_{\mathbf{x} \star}^{\sharp}\right)^{-1} \chi_{\text {trans }}^{\frac{1}{2}}\right)^{\frac{1}{2}} \chi_{\text {trans }}^{-\frac{1}{2}}+\left(\mathcal{H}_{\mathbf{x} \star}^{\sharp}\right)^{-1}=\left(\mathcal{H}_{\mathbf{x} \star}^{\sharp}\right)^{-1}-\mathcal{B}_{\mathbf{x}}^{\star} .
\end{aligned}
$$

[6] J. Armstrong, Y. A. Sekercioglu and A. Neild, "Visible Light Positioning: A Roadmap for international Standardization", IEEE Communication Magzine, Vol.51, No.12, 2013, pp.68-73.

[7] S. Y. Jung, S. Hann, and C.-S. Park. ”TDOA-based optical wireless indoor localization using LED ceiling lamps." IEEE Transactions on Consumer Electronics, Vol.57, No.4, 2011, pp.1592-1597.

[8] C. Amini, A. Taherpour, T. Khattab and S. Gazor, "Theoretical accuracy analysis of indoor visible light communication positioning system based on time-of-arrival," 2016 IEEE Canadian Conference on Electrical and Computer Engineering (CCECE), Vancouver, BC, 2016, pp. 1-5.

[9] K. Panta and J. Armstrong, "Indoor localization using white LEDs," Electron. Lett., vol. 48, no. 4, pp. 228-230, Feb. 2012.

[10] X. Zhang, J. Duan, Y. Fu and A. Shi, "Theoretical Accuracy Analysis of Indoor Visible Light Communication Positioning System Based on Received Signal Strength Indicator," Journal of Lightwave Technology, Vol.32, No.21, 2014, pp.4180-4186.

[11] L. Yin, X. Wu and H. Haas, "Indoor Visible Light Positioning with Angle Diversity Transmitter." 2015 IEEE 82nd Vehicular Technology Conference (VTC Fall). IEEE, 2015.

[12] Y. S. Eroglu, I. Guvenc, N. Pala and M. Yuksel, "AOA-based localization and tracking in multi-element VLC systems," 2015 IEEE 16th Annual Wireless and Microwave Technology Conference (WAMICON), Cocoa Beach, FL, 2015, pp. 1-5.

[13] A. Sahin, Y.S. Eroglu, I. Guvenc, N. Pala, and M. Yuksel, "Accuracy of AOA-based and RSS-based 3D localization for visible light communications." Vehicular Technology Conference (VTC Fall), 2015 IEEE 82nd. IEEE, 2015.

[14] H. Kim, D. Kim, S. Yang, Y. Son, and S. Han, "An Indoor Visible Light Communication Positioning System Using a RF Carrier Allocation Technique," J. Lightw. Technol., Vol.31, No.1, 2013, pp. 134-144.

[15] C. Sertthin, T. Ohtsuki, and M. Nakagawa, "6-Axis Sensor Assisted Low Complexity High Accuracy-Visible Light Communication Based Indoor Positioning System," IEICE Trans. Commun., Vol.E93-B, No.11, 2010, pp. 2879-2891.

[16] Z. Zhou, M. Kavehrad, and P. Deng, "Indoor Positioning Algorithm using Light-Emitting Diode Visible Light Communications," Optical Engineering, 2012, 51(8), 085009-1.

[17] T. H. Do, and M. Yoo, "An in-depth survey of visible light communication based positioning systems," Sensors, 2015, 16(5), 678.

[18] B. Zhou, Q. Chen, P. Xiao and L. Zhao, "On the Spatial Error Propagation Characteristics of Cooperative Localization in Wireless Networks," IEEE Trans. Vehi. Tech., Vol.66, No.2, 2017, pp.1647-1658.

[19] B. Zhou, Q. Chen, and P. Xiao. "The error propagation analysis of the received signal strength-based simultaneous localization and tracking in wireless sensor networks." IEEE Transactions on Information Theory 63.6 (2017): 3983-4007.

[20] B. Zhou, Q. Chen, H. Wymeersch, P. Xiao and L. Zhao, "Variational Inference-based Positioning with Nondeterministic Measurement Accuracies and Reference Location Errors," IEEE Transactions on Mobile Computing. Vol.16, No. 10, Oct, 2017, pp.2955-2969
[21] B. Zhou and Q. Chen, "On the Particle-Assisted Stochastic Search Mechanism in Wireless Cooperative Localization," IEEE Transactions on Wireless Communications, 2016, Vol.15, No.7, pp. 4765-4777.

[22] M. F. Keskin, A. D. Sezer, and S. Gezici, "Optimal and robust power allocation for visible light positioning systems under illumination constraints." IEEE Transactions on Communications, 67.1, (2019): 527-542.

[23] M. Z. Win, W. Dai, Y. Shen, G. Chrisikos, and H. V. Poor, "Network operation strategies for efficient localization and navigation." Proceedings of the IEEE, 106.7, (2018): 1224-1254.

[24] W. H. Dai, Y. Shen, and M. Z. Win, "A computational geometry framework for efficient network localization." IEEE Transactions on Information Theory, 64.2 (2018): 1317-1339.

[25] Y. Shen, W. Dai, and M. Z. Win, "Power optimization for network localization." IEEE/ACM Transactions on Networking, 22.4 (2014): 13371350.

[26] Y. Shen, W. Dai, and M. Z. Win, "Distributed power allocation for cooperative wireless network localization." IEEE Journal on Selected Areas in Communications, 33.1 (2015): 28-40.

[27] Y. Shen, W. Dai, and M. Z. Win, "Energy-efficient network navigation algorithms." IEEE Journal on Selected Areas in Communications, 33.7 (2015): 1418-1430.

[28] Y. Shen, and M. Z. Win. "Fundamental limits of wideband localizationPart I: A general framework." IEEE Transactions on Information Theory, 56.10 (2010): 4956-4980.

[29] Y. Shen, H. Wymeersch and M. Z. Win, "Fundamental limits of wideband localization-Part II: Cooperative Networks." IEEE Trans. Inform. Theory, vol.56, no.10, 2010, pp.4956-4979.

[30] M. Z. Win, Y. Shen, and W. Dai. "A theoretical foundation of network localization and navigation." Proceedings of the IEEE, 106.7 (2018): 1136-1165.

[31] E. Steinmetz, R. Emardson, F. Brännström, and H. Wymeersch, 'Theoretical Limits on Cooperative Positioning in Mixed Traffic." IEEE Access, 7 (2019): 49712-49725.

[32] H. Steendam, T. Q. Wang and J. Armstrong, "Cramer-Rao bound for indoor visible light positioning using an aperture-based angular-diversity receiver," 2016 IEEE International Conference on Communications (ICC), Kuala Lumpur, 2016, pp. 1-6.

[33] T. Q. Wang, Y. A. Sekercioglu, A. Neild and J. Armstrong, "Position Accuracy of Time-of-Arrival Based Ranging Using Visible Light With Application in Indoor Localization Systems," Journal of Lightwave Technology, Vol.31, No.20, pp.3302-3308, Oct.15, 2013.

[34] M. F. Keskin, S. Gezici, and O. Arikan. "Direct and two-step positioning in visible light systems." IEEE Transactions on Communications, 2018, No.66 Vol.1, pp.239-254.

[35] M. F. Keskin, A. D. Sezer, and S. Gezici, "Localization via visible light systems." Proceedings of the IEEE, 106.6 (2018): 1063-1088.

[36] S. Rajagopal, R. D. Roberts, and S.-K. Lim. "IEEE 802.15. 7 visible light communication: modulation schemes and dimming support." IEEE Communications Magazine, Vol.50 No.3, 2012, pp.72-82. 
[37] H. Elgala, R. Mesleh and H. Haas, "Indoor optical wireless communication: potential and state-of-the-art," IEEE Communication Magazine, Vol.49, No.9, 2011, pp.56-62.

[38] S. M. Kay, Fundamentals of Statistical Signal Processing, Vol. 2: Detection theory. Prentice Hall PTR, 1998.

[39] T. Cover and J. Thomas, Elements of Information Theory, New York: John Wiley and Sons, Inc., 1991.

[40] S. N. Diggavi and T. M. Cover, 'Is maximum entropy noise the worst?", Proceedings of IEEE International Symposium on Information Theory, Ulm, 1997, pp.278.

[41] Y. Qi, H. Kobayashi, and H. Suda, "On time-of-arrival positioning in a multipath environment," IEEE Transactions on Vehicular Technology, 2006, vol. 55, no. 5, pp. 1516-1526.

[42] S. Gezici and Z. Sahinoglu, "Ranging in a single-input multiple-output (SIMO) system," IEEE Communications Letters, vol. 12, no. 3, 2008, pp. 197-199.

[43] M. Z. Win, A. Conti, S. Mazuelas, Y. Shen, W. M. Gifford, D. Dardari, M. Chiani, "Network localization and navigation via cooperation." IEEE Communications Magazine, 49.5 (2011): 56-62.

[44] H. Wymeersch, J. Lien, and M. Z. Win. "Cooperative localization in wireless networks." Proceedings of the IEEE, 97.2 (2009): 427-450.

[45] C. Taylor, A. Rahimi, J. Bachrach, H. Shrobe, and A. Gure, "Simultaneous localization, calibration, and tracking in an ad hoc sensor network." Proceedings of the 5th international conference on Information processing in sensor networks. ACM, 2006: 27-33.

[46] K. Friston, J. Mattout, N. Trujillo-Barreto, J. Ashburner, and W. Pennya, "Variational free energy and the Laplace approximation." NeuroImage, ELSEVIER, vol.34, no.1, 2006, pp.220-234.

[47] B. Zhou, V. Lau, Q. Chen, and Y. Cao, "Simultaneous Positioning and Orientating (SPAO) for Visible Light Communications: Algorithm Design and Performance Analysis," IEEE Transactions on Vehicular Technology, Vol. 67, No. 12, 2018, PP. 11790-11804

[48] P. Luo, Z. Ghassemlooy, H. Le Minh, E. Bentley, A. Burton, and X. Tang, "Fundamental analysis of a car to car visible light communication system." Communication Systems, Networks \& Digital Signal Processing (CSNDSP), 2014 9th International Symposium on. IEEE, 2014.

[49] X. Chen, and M. Jiang. "Adaptive statistical Bayesian MMSE channel estimation for visible light communication." IEEE Transactions on Signal Processing 65.5 (2017): 1287-1299.

[50] S. W. Ho, A. A. Saed, L. Lai, and C. W. Sung, "Coding and Bounds for Channel Estimation in Visible Light Communications and Positioning." IEEE Journal on Selected Areas in Communications 36.1 (2018): 34-44.

[51] M. Yasir, S. W. Ho and B. N. Vellambi, "Indoor localization using visible light and accelerometer," 2013 IEEE Global Communications Conference (GLOBECOM), Atlanta, GA, 2013, pp.3341-3346.

[52] B. Zhou, Q. Chen, T. J. Li, and P. Xiao, "Online variational Bayesian filtering-based mobile target tracking in wireless sensor networks." Sensors, 14.11 (2014): 21281-21315.

[53] S. Boyd, and Lieven Vandenberghe. Convex optimization. Cambridge university press, 2004.

[54] J. Mairal. "Stochastic majorization-minimization algorithms for largescale optimization." Advances in Neural Information Processing Systems. 2013, pp. 2283-2291

[55] A. Liu, V. K. N. Lau, and M.-J. Zhao. "Stochastic Successive Convex Optimization for Two-timescale Hybrid Precoding in Massive MIMO." IEEE Journal of Selected Topics in Signal Processing, (2018).

[56] M. Yasir, S.-W. Ho, and B. N. Vellambi. "Indoor positioning system using visible light and accelerometer." Journal of Lightwave Technology 32.19 (2014): 3306-3316.

[57] J. Kahn and J. Barry, "Wireless Infrared Communications," Proc. IEEE, Vol.85, No.2, 1997, pp. 265-298. 